\title{
FINITE JET DETERMINATION OF HOLOMORPHIC MAPPINGS AT THE BOUNDARY*
}

\author{
PETER EBENFELT ${ }^{\dagger}$
}

0. Introduction. A classical theorem of $\mathrm{H}$. Cartan $([\mathrm{HCa}])$ states that an automorphism $f$ of a bounded domain $D \subset \mathbb{C}^{N}$ is completely determined by its 1-jet, i.e. its value and derivatives of order one, at any point $Z_{0} \in D$. If $D$, in addition, is assumed to be smoothly $\left(C^{\infty}\right)$ bounded and strictly pseudoconvex, then by Fefferman's theorem $[\mathrm{Fe}]$ any such automorphism extends smoothly to the boundary $\partial D$ as an automorphism $\partial D \rightarrow \partial D$. It is then natural to ask: is $f$ completely determined by a finite jet at a boundary point $p \in \partial D$ ? An affirmative answer to this question, when $D$ is strictly pseudoconvex, follows from the work of Chern and Moser [CM] (see also E. Cartan [ECa1-2] for the case $N=2$, and Tanaka [T1-2]). Indeed, the following local version of Cartan's theorem is a consequence of their work. Any holomorphic mapping which is defined locally on one side of a smooth, Levi nondegenerate real hypersurface $M \subset \mathbb{C}^{N}$ and extends smoothly to $M$, sending $M$ diffeomorphically into another smooth real hypersurface $M^{\prime} \subset \mathbb{C}^{N}$, is completely determined by its 2-jet at a point $p \in M$. Observe that the conclusion is nontrivial even in the strictly pseudoconcave case when the mapping extends holomorphically to a full neighborhood of $p$.

The main objective of the present paper is to extend the above mentioned local result to a more general class of real hypersurfaces (Theorem 1 below). We should point out that the result for Levi nondegenerate hypersurfaces follows from the construction of a unique Cartan connection on a certain principal $G$-bundle over such a hypersurface. There is no analogue of this construction in the more general situation considered in this paper.

Let $M \subset \mathbb{C}^{N}$ be a smooth real hypersurface and assume that $M$ is defined locally near a point $p_{0} \in M$ by the equation $\rho(z, \bar{z})=0$, where $\rho$ is a smooth function with $\rho\left(p_{0}, \bar{p}_{0}\right)=0$ and $d \rho\left(p_{0}, \bar{p}_{0}\right) \neq 0$. Let $L_{\overline{1}}, \ldots, L_{\bar{n}}$, with $n=N-1$, be a basis for the $\mathrm{CR}$ vector fields on $M$. We shall say that $M$ is $k_{0}$-nondegenerate at $p_{0}$ if

$$
\operatorname{Span}\left\{L^{\bar{\alpha}} \rho_{z}\left(p_{0}, \bar{p}_{0}\right):|\alpha| \leq k_{0}\right\}=\mathbb{C}^{N}
$$

where $\rho_{z}:=\left(\partial_{z_{j}} \rho\right)_{1 \leq j \leq N}, \partial_{z_{j}}:=\partial / \partial z_{j}$, and standard multi-index notation for differential operators is used i.e. $L^{\bar{\alpha}}:=L_{\overline{1}}^{\bar{\alpha}_{1}} \ldots L_{\bar{n}}^{\bar{\alpha}_{n}}$. This nondegeneracy condition will be given in a different, but equivalent, form in terms of the intrinsic geometry of $M$ in the next section. The reader is referred to the book [BER3] for basic material on real submanifolds in complex space and CR structures, and further discussion of various nondegeneracy conditions (see also $\S 1$ of the present paper). We mention here only that Levi nondegeneracy at a point $p_{0} \in M$ is equivalent to 1-nondegeneracy. Our main result is the following.

* Received October 23, 2000; accepted for publication February 8, 2001.

$\dagger$ Department of Mathematics, Royal Institute of Technology, 10044 Stockholm, Sweden. Current Add: Department of Mathematics, University of California at San Diego, La Jolla, CA 92093 (pebenfel@math.ucsd.edu, ebenfelt@math.kth.se). The author is supported in part by a grant from the Swedish Natural Science ResearchCouncil. 
TheOREM 1. Let $M, M^{\prime} \subset \mathbb{C}^{N}$ be smooth $\left(C^{\infty}\right)$ real hypersurfaces. Let $f, g: U \rightarrow$ $\mathbb{C}^{N}$, where $U \subset \mathbb{C}^{N}$ is an open connected subset with $M$ in its boundary, be holomorphic mappings which extend smoothly to $M$ and send $M$ diffeomorphically into $M^{\prime}$. If $M$ is $k_{0}$-nondegenerate at a point $p_{0} \in M$ and

$$
\left(\partial_{z}^{\alpha} f\right)\left(p_{0}\right)=\left(\partial_{z}^{\alpha} g\right)\left(p_{0}\right), \quad \forall \alpha \in \mathbb{Z}_{+}^{N}:|\alpha| \leq 2 k_{0},
$$

then $f \equiv g$ in $U$.

Finite jet determination of holomorphic mappings sending one real submanifold into another has attracted much attention in recent years. We mention here the papers [BER1-2, 4-5], [L], [Han1-2], [Hay], [Z]. The reader is also referred to the survey article [BER6] for a more detailed history. However, in all the above mentioned papers, it is either assumed that $M$ and $M^{\prime}$ are real-analytic (which will imply that all mappings $f$ extend holomorphically to some neighborhood of $M$ ), or the conclusion is that the formal power series of the mapping $f$ is determined by a finite jet (see [BER4], [L]). Theorem 1 appears to be, to the best of the author's knowledge, the first finite determination result, since the work of Chern and Moser mentioned above, which applies to merely smooth hypersurfaces and smooth mappings. We should mention that if $M$ and $M^{\prime}$ are real-analytic, then the conclusion of Theorem 1 was proved in [BER2] (cf. also [Han1] and [Z]). A related notion is that of unique continuation the boundary for holomorphic mappings. A unique continuation principle is said to hold for a class of mappings at a point $p$ if any mapping from this class which agrees with the constant mapping to infinite order at $p$ is necessarily constant. (Observe that, due to the nonlinear nature of mapping problems, a unique continuation principle for a class of mappings into a manifold does not imply that two mappings, in this class, which agree to infinite order are necessarily the same.) We shall not address this problem further here. We mention the papers $[\mathrm{ABR}],[\mathrm{BR}],[\mathrm{BL}],[\mathrm{CR}],[\mathrm{E} 1],[\mathrm{HK}]$, and refer the interested reader to these papers for further information.

The proof of Theorem 1 is based on Theorem 2 below, and a result from [BER4], alluded to above, which asserts that, under the assumptions of Theorem 1 , the jet of $f$ at $p_{0}$ of any order is completely determined by its $2 k_{0}$-jet. The proof of Theorem 1 is given at the end of $\S 3$.

Our second result, which is the basis for Theorem 1 above, states, loosely speaking, that given two suitably nondegenerate real hypersurfaces, there is a system of differential equations, which is complete in a certain sense, such that any CR diffeomorphism $f: M \rightarrow M^{\prime}$ must satisfy this system. The idea to look for such a differential system goes back to the work of E. Cartan and Chern-Moser mentioned above. The approach was further developed in the work of Han. To formulate the result more precisely, we need to fix some notation. Let us denote by $J^{k}\left(M, M^{\prime}\right)_{\left(p, p^{\prime}\right)}$ the space of $k$-jets at $p \in M$ of smooth mappings $f: M \rightarrow M^{\prime}$ with $f(p)=p^{\prime} \in M^{\prime}$. Given coordinate systems $x=\left(x_{1}, \ldots, x_{2 N-1}\right)$ and $x^{\prime}=\left(x_{1}^{\prime}, \ldots, x_{2 N-1}^{\prime}\right)$ on $M$ and $M^{\prime}$ near $p$ and $p^{\prime}$, respectively, there are natural coordinates $\lambda^{k}:=\left(\lambda_{i}^{\beta}\right)$, where $1 \leq i \leq 2 N-1$ and $\beta \in \mathbb{Z}_{+}^{2 N-1}$ with $1 \leq|\beta| \leq k$, on $J^{k}\left(M, M^{\prime}\right)_{\left(p, p^{\prime}\right)}$ in which the $k$-jet at $p$ of a smooth mapping $f: M \rightarrow M^{\prime}$ is given by $\lambda_{i}^{\beta}=\left(\partial_{x}^{\beta} f_{i}\right)(p), 1 \leq|\beta| \leq k$ and $1 \leq i \leq 2 N-1$.

Theorem 2. Let $M, M^{\prime} \subset \mathbb{C}^{N}$ be smooth $\left(C^{\infty}\right)$ real hypersurfaces. Assume that $M$ is $k_{0}$-nondegenerate at a point $p_{0}$. Let $f^{0}: M \rightarrow M^{\prime}$ be a smooth $C R$ diffeomorphism. Then, for any multi-index $\alpha \in \mathbb{Z}_{+}^{2 N-1}$ with $|\alpha|=k_{0}^{3}+k_{0}^{2}+k_{0}+2$ and any $j=1, \ldots, 2 N-1$, there are smooth functions $r_{j}^{\alpha}\left(\lambda^{k} ; x^{\prime}\right)(x)$ on $U$, where 
$k:=k_{0}^{3}+k_{0}^{2}+k_{0}+1$ and $U \subset J^{k}\left(M, M^{\prime}\right)_{\left(p_{0}, p_{0}^{\prime}\right)} \times M \times M^{\prime}$ is an open neighborhood of $\left(\left(\partial_{x}^{\beta} f^{0}\right)(0), f^{0}\left(p_{0}\right), p_{0}\right)$, such that

$$
\partial_{x}^{\alpha} f_{j}=r_{j}^{\alpha}\left(\partial_{x}^{\beta} f ; f\right), \quad \forall|\alpha|=k_{0}^{3}+k_{0}^{2}+k_{0}+2, j=1 \ldots, 2 N-1,
$$

where $1 \leq|\beta| \leq k$, for every smooth CR diffeomorphism $f: V \rightarrow M^{\prime}$, where $V \subset$ $M$ is some open neighborhood of $p_{0}$, with $\left(\left(\partial_{x}^{\beta} f\right)(0), f\left(p_{0}\right), p_{0}\right) \in U$. Moreover, the functions $r_{j}^{\alpha}$ are rational in $\lambda^{k} \in J^{k}\left(M, M^{\prime}\right)_{\left(p_{0}, p_{0}^{\prime}\right)}$; here, $x=\left(x_{1}, \ldots, x_{2 N-1}\right)$ and $x^{\prime}=\left(x_{1}^{\prime}, \ldots, x_{2 N-1}^{\prime}\right)$ are any local coordinate systems on $M$ and $M^{\prime}$ near $p_{0}$ and $f^{0}\left(p_{0}\right)$, respectively, and $f_{i}:=f \circ x_{i}^{\prime}$.

Similar results for real-analytic hypersurfaces can also be found in [Han1-2] and [Hay]. The idea behind the proof of Theorem 2 is to consider the tangent mapping $d f: \mathbb{C} T M \rightarrow \mathbb{C} T M^{\prime}$ and derive differential equations for $d f$ using properties of a sequence of invariant tensors (generalized Levi forms) which were developed in the author's paper [E3]. The proof of Theorem 2 is given in $\S 3$.

We conclude this introduction by giving two applications of Theorems 1 and 2 . For this, we need some more notation. A smooth real vector field $X$ on $M$ is called an infinitesimal CR automorphism if the local 1-parameter group of diffeomorphisms, $\exp t X$, generated by $X$ is a local group of CR diffeomorphisms (see e.g. [BER2] or [S1-2]). The set of infinitesimal CR automorphisms, defined near $p \in M$, forms a vector space over $\mathbb{R}$ denoted by aut $(M, p)$. We shall give a sufficient condition on $M$ at a point $p$ for $\operatorname{dim}_{\mathbb{R}}$ aut $(M, p)<\infty$. A smooth real hypersurface $M \subset \mathbb{C}^{N}$ is called (formally) holomorphically degenerate at $p \in M$, if there exists a formal holomorphic vector field

$$
Y=\sum_{j=1}^{N} a_{j}(z) \partial_{z_{j}}
$$

where the $a_{j}(z)$ are formal power series in $z-p$, which is tangent to $M$, i.e. such that the Taylor series at $p$ of a defining function $\rho(z, \bar{z})$ for $M$ divides $(Y \rho)(z, \bar{z})$ in the ring of formal power series in $(z-p, \bar{z}-\bar{p})$. Being holomorphically nondegenerate (i.e. the opposite of being degenerate) at a point is a strictly weaker condition than that of being $k$-nondegenerate for some integer $k$. (See [BER3, Chapter XI] for a more detailed description of the relationship between the two notions). Also, recall that $M$ is said to be minimal at $p \in M$ (in the sense of Tumanov and Trepreau) if $M$ does not contain a complex hypersurface through $p$.

Theorem 3. Let $M \subset \mathbb{C}^{N}$ be a smooth $\left(C^{\infty}\right)$ real hypersurface which is holomorphically nondegenerate and minimal at $p_{0}$. Then,

$$
\operatorname{dim}_{\mathbb{R}} \operatorname{aut}\left(M, p_{0}\right) \leq(2 N-1)\left(\begin{array}{l}
4 N-3 \\
2 N-2
\end{array}\right)
$$

A real-analytic hypersurface $M$ is said to be holomorphically degenerate at $p \in M$ if there exists a holomorphic vector field, i.e. a vector field of the form (0.4) with the $a_{j}(z)$ holomorphic, tangent to $M$ near $p$. This definition turns out to be equivalent to the one given in the smooth category above (i.e. using formal vector fields) for a real-analytic hypersurface (see [BER3, Proposition 11.7.4]). Stanton [S2] proved that $\operatorname{dim}_{\mathbb{R}} \operatorname{hol}(M, p)<\infty$ for a real-analytic hypersurface $M$, where $\operatorname{hol}(M, p)$ denotes the subspace of $\operatorname{aut}(M, p)$ consisting of those infinitesimal CR automorphisms which are 
real-analytic, if and only if $M$ is holomorphically nondegenerate at $p$. The corresponding statement (as well as results for higher codimensional real-analytic submanifolds) for $\operatorname{aut}(M, p)$, with $M$ real-analytic, was proved in [BER2]. In contrast to the realanalytic case, the condition of (formal) holomorphic nondegeneracy is not necessary in Theorem 3. A real smooth hypersurface $M$ in $\mathbb{C}^{2}$ which is holomorphically degenerate and minimal at 0 , but everywhere Levi nondegenerate outside 0 is given in [BER3, Example 11.7.29]. The fact that $M$ is Levi nondegenerate outside 0 can be seen to imply (see the concluding remarks in $\S 4.2$ ) that $\operatorname{dim}_{\mathbb{R}}$ aut $(M, 0)$ satisfies the bound in (0.5). However, if there exists a vector field

$$
Y=\sum_{j=1}^{N} a_{j}(z, \bar{z}) \partial_{z_{j}},
$$

where the $a_{j}(z, \bar{z})$ are smooth functions whose restrictions to $M$ are CR, tangent to $M$ near $p$, then the arguments in [S2] easily show that $\operatorname{dim}_{\mathbb{R}} \operatorname{aut}(M, 0)=\infty$. This discrepancy is addressed further in $\S 4.2$. The proof of Theorem 3 is given in $\S 3$.

For our final result, we shall denote by $\operatorname{Aut}(M, p)$ the stability group of $M$ at $p \in M$, i.e. the group of germs at $p$ of local CR diffeomorphisms $f: V \rightarrow M$, where $V \subset M$ is some open neighborhood of $p$, with $f(p)=p$. If $M$ is $k_{0}$-nondegenerate at $p_{0}$, then, by Theorem 1 , the jet mapping $j_{p}^{2 k_{0}}$ sends $\operatorname{Aut}\left(M, p_{0}\right)$ injectively into the jet group $G^{2 k_{0}}\left(\mathbb{C}^{N}\right)_{p_{0}} \subset J^{2 k_{0}}\left(\mathbb{C}^{N}, \mathbb{C}^{N}\right)_{\left(p_{0}, p_{0}\right)}$, which consists of those jets that are invertible at $p_{0}$. We shall show that the elements of $\operatorname{Aut}\left(M, p_{0}\right)$ depend smoothly on their $2 k_{0}$-jets at $p_{0}$. More precisely, we have the following result.

THEOREM 4. Let $M \subset \mathbb{C}^{N}$ be a smooth $\left(C^{\infty}\right)$ real hypersurface which is $k_{0}$ nondegenerate at $p_{0} \in M$. Then, the jet mapping

$$
j^{2 k_{0}}: \operatorname{Aut}\left(M, p_{0}\right) \rightarrow G^{2 k_{0}}\left(\mathbb{C}^{N}\right)_{p_{0}}
$$

is injective and, for every $f^{0} \in \operatorname{Aut}\left(M, p_{0}\right)$, there exist an open neighborhood $U_{0}$ of $j_{p_{0}}^{2 k_{0}}\left(f^{0}\right)$ in $G^{2 k_{0}}\left(\mathbb{C}^{N}\right)_{p_{0}}$, an open neighborhood $V_{0}$ of $p_{0}$ in $M$, and a smooth $\left(C^{\infty}\right)$ mapping $F: U_{0} \times V_{0} \rightarrow M$ such that

$$
F\left(j_{p_{0}}^{2 k_{0}}(f), \cdot\right)=f
$$

for every $f \in \operatorname{Aut}\left(M, p_{0}\right)$ with $j_{p_{0}}^{2 k_{0}}(f) \in U_{0}$.

For real-analytic hypersurfaces, the result in Theorem 4 (with real-analytic dependence) was proved in [BER1]. (See [BER4] for the higher codimensional case; $\mathrm{cf}$. also [Z].).

Acknowledgement. The author would like to thank B. Lamel and D. Zaitsev for many helpful comments and discussions on a preliminary version of this paper.

1. Preliminaries. A real hypersurface $M \subset \mathbb{C}^{N}$ inherits a $\mathrm{CR}$ structure $\mathcal{V}:=$ $T^{0,1} \mathbb{C}^{N} \cap \mathbb{C} T M$ from the ambient complex space $\mathbb{C}^{N}$. (Here, $T^{0,1} \mathbb{C}^{N}$ denotes the usual bundle of $(0,1)$ vectors in $\mathbb{C}^{N}$.) In this section, we shall consider abstract, not necessarily embedded (or integrable), CR structures. At the end of this section, we shall again specialize to embedded hypersurfaces, which substantially simplifies some of the computations in subsequent sections. The reader is referred to the concluding remarks in $\S 4$ for a brief discussion of the abstract case.

Let $M$ be a smooth $\left(C^{\infty}\right)$ manifold with a CR structure $\mathcal{V} \subset \mathbb{C} T M$. Recall that this means that $\mathcal{V}$ is a formally integrable subbundle (the commutator of two sections 
of $\mathcal{V}$ is again a section of $\mathcal{V}$ ) such that $\mathcal{V}_{p} \cap \overline{\mathcal{V}}_{p}=\{0\}$ for every $p \in M$. Sections of the CR bundle are called $C R$ vector fields. We shall denote by $n \geq 1$ the CR dimension of the CR manifold $M$, which by definition is the complex fiber dimension of $\mathcal{V}$, and we shall assume that the $C R$ structure is of hypersurface type, i.e. that $\operatorname{dim}_{\mathbb{R}} M=2 n+1$. The reader is referred to [BER3] for an introduction to CR structures.

We define two subbundles $T^{0} M \subset T^{\prime} M \subset \mathbb{C} T^{*} M$ as follows

$$
T^{0} M:=(\mathcal{V} \oplus \overline{\mathcal{V}})^{\perp} . \quad T^{\prime} M=\mathcal{V}^{\perp},
$$

where $A^{\perp} \subset \mathbb{C} T^{*} M$, for a subset $A \subset \mathbb{C} T M$. denotes the union over $p \in M$ of the set of covectors at $p$ annihilating every vector in $A_{p}$. Real nonvanishing sections of $T^{0} M$ are called characteristic forms and sections of $I^{\prime} . M$ are called holomorphic forms. Thus, characteristic forms are in particular holomorphic forms.

We shall give an alternative definition of $k_{0}$-nondegeneracy, as defined in the introduction, in terms of the intrinsic geometry of .M. This definition appeared in [E2]. For a holomorphic form $\omega$, the Lie derivative with respect to a $\mathrm{CR}$ vector field $X$ is given by

$$
\left.\mathcal{L}_{X} \omega=X\right\lrcorner d \omega .
$$

where $\lrcorner$ denotes the interior product, or contraction, and $d$ denotes exterior differentiation. For $p \in M$, define the subspaces

$$
T_{p}^{0} M:=E_{0}(p) \subset E_{1}(p) \subset \ldots \subset E_{k}(p) \subset \ldots \subset T_{p}^{\prime} M
$$

by letting $E_{k}(p)$ be the linear span (over $\mathbb{C}$ ) of the holomorphic covectors

$$
\left(\mathcal{L}_{X_{k}} \ldots \mathcal{L}_{X_{1}} \theta\right)(p)
$$

where $X_{1}, \ldots, X_{k}$ range over all $\mathrm{CR}$ vector fields and $\theta$ over all characteristic forms near $p . M$ is called finitely nondegenerate at $p \in M$ if $E_{k}(p)=T_{p}^{\prime} M$ for some $k$. More precisely, we say that $M$ is $k_{0}$-nondegenerate at $p$ if

$$
E_{k_{0}-1}(p) \varsubsetneqq E_{k_{0}}(p)=T_{p}^{\prime}: M
$$

For an argument showing that this definition coincides with that given for embedded hypersurfaces in the introduction, the reader is referred to [BER3] (see also [E2]). For each $k$, set

$$
F_{k}(p)=\overline{\mathcal{V}}_{p} \cap E_{k}(p)^{-}
$$

It was shown in [E3] that the mapping

$$
\left(X_{1}, \ldots, X_{k}, Y, \theta\right) \mapsto\left\langle\left(\mathcal{L}_{X_{k}} \ldots \mathcal{L}_{X_{1}} \theta\right)(p), Y(p)\right\rangle
$$

defines a multi-linear mapping

$$
\mathcal{V}_{p} \times \ldots \mathcal{V}_{p} \times F_{k-1}(p) \times T_{p}^{0}: M \rightarrow \mathbb{C}
$$

which is symmetric in the first $k$ positions. The tensor so defined for $k=1$ coincides with the classical Levi form, and the space $F_{1}(0)$ is the Levi nullspace.

Let us fix a distinguished point on $M$ denoted by $0 \in M$. We choose a basis $L_{1}, \ldots, L_{n}$ of the sections $C^{\infty}(U, \overline{\mathcal{V}})$, where $U \subset M$ is some sufficiently small neighborhood of 0 , adapted to the filtration

$$
\overline{\mathcal{V}}_{0}=F_{0}(0) \supset F_{1}(0) \supset \ldots \supset F_{k}(0) \supset \ldots \supset\{0\}
$$


in the following way. Observe that the sequence of subspaces $F_{k}(0)$ stabilizes at a smallest subspace $F_{k_{0}}(0)$, which equals $\{0\}$ if and only if $M$ is $k_{0}$-nondegenerate at 0 . Let $r_{k}=n-\operatorname{dim}_{\mathbb{C}} F_{k}(0)$ and choose $L_{1}, \ldots, L_{n}$ so that $L_{r_{k}+1}(0), \ldots L_{n}(0)$ spans $F_{k}(0)$ for $k=0,1, \ldots, k_{0}$. We shall use the following conventions for indices. For $j=1,2, \ldots$, Greek indices $\alpha^{(j)}, \beta^{(j)}$, etc., will run over the set $\left\{1, \ldots, r_{j-1}\right\}$ and small Roman indices $a^{(j)}, b^{(j)}$, etc., over $\left\{r_{j-1}+1, \ldots, n\right\}$. Capital Roman indices $A, B$, etc., will run over $\{1, \ldots, n\}$.

Now, choose also a characteristic form $\theta$ on $M$ near 0 . We write

$$
h_{\bar{A}_{1} \ldots \bar{A}_{k} B}:=\left\langle\mathcal{L}_{\bar{A}_{k}} \ldots \mathcal{L}_{\bar{A}_{1}} \theta, L_{B}\right\rangle,
$$

where $\mathcal{L}_{\bar{A}}:=\mathcal{L}_{L_{\bar{A}}}$ and $L_{\bar{A}}:=\overline{L_{A}}$. Note that $\left(h_{\bar{A}_{1} \ldots \bar{A}_{k} a^{(k)}}(0)\right)$ represents the tensor defined by (1.7) relative to the bases $L_{\bar{A}}(0), L_{a^{(k)}}(0)$, and $\theta(0)$ of $\mathcal{V}_{0}, F_{k}(0)$, and $T_{0}^{0} M$, respectively.

Let $T$ be a vector field near 0 such that $T, L_{A}, L_{\bar{A}}$ form a basis for $C^{\infty}(U, \mathbb{C} T M)$. Let $\theta, \theta^{A}, \theta^{\bar{A}}$ be the dual basis for $C^{\infty}\left(U, \mathbb{C} T^{*} M\right)$. Note that, for each $k=1, \ldots, k_{0}$, the covectors $\theta(0), \theta^{\alpha^{(k)}}(0)$ form a basis for $E_{k}(0)$. For brevity, we introduce the functions

$$
h_{\bar{A}_{1} \ldots \bar{A}_{k}}:=\left\langle\mathcal{L}_{\bar{A}_{k}} \ldots \mathcal{L}_{\bar{A}_{1}} \theta, T\right\rangle
$$

and also

$$
\begin{array}{cl}
R_{\bar{A} B}^{C}:=\left\langle d \theta^{C}, L_{\bar{A}} \wedge L_{B}\right\rangle, & R_{D B}^{C}:=\left\langle d \theta^{C}, L_{D} \wedge L_{B}\right\rangle \\
R_{\bar{A}}^{C}:=\left\langle d \theta^{C}, L_{\bar{A}} \wedge T\right\rangle, & R_{B}^{C}:=\left\langle d \theta^{C}, T \wedge L_{B}\right\rangle .
\end{array}
$$

The following identity is useful.

Lemma 1.13. For any nonnegative integer $k$, and indices $A_{1}, \ldots, A_{k}, C, D \in$ $\{1, \ldots, n\}$, the following identity holds

$$
h_{\bar{A}_{1} \ldots \bar{A}_{k} \bar{C} D}=L_{\bar{C}} h_{\bar{A}_{1} \ldots \bar{A}_{k} D}+h_{\bar{A}_{1} \ldots \bar{A}_{k} B} R_{\bar{C} D}^{B}+h_{\bar{A}_{1} \ldots \bar{A}_{k}} h_{\bar{C} D}
$$

Proof. Recall that $\mathcal{L}_{\bar{A}_{k}} \ldots \mathcal{L}_{\bar{A}_{1}} \theta$ is a holomorphic 1-form and, by the definitions $(1.10-11)$,

$$
\mathcal{L}_{\bar{A}_{k}} \ldots \mathcal{L}_{\bar{A}_{1}} \theta=h_{\bar{A}_{1} \ldots \bar{A}_{k} D} \theta^{D}+h_{\bar{A}_{1} \ldots \bar{A}_{k}} \theta .
$$

Here, and for the remainder of this paper, we use the summation convention which states that an index appearing in both a sub- and superscript is summed over; e.g. $h_{D} \theta^{D}=\sum_{D} h_{D} \theta^{D}$. We also have, by the definition of the interior product,

$$
h_{\bar{A}_{1} \ldots \bar{A}_{k} \bar{C} D}=\left\langle d \mathcal{L}_{\bar{A}_{k}} \ldots \mathcal{L}_{\bar{A}_{1}} \theta, L_{\bar{C}} \wedge L_{D}\right\rangle .
$$

The identity (1.14) follows by applying the exterior derivative $d$ to (1.15) and substituting in (1.16).

Define $\ell_{0}$ to be the smallest integer $\ell$ for which

$$
\left\{\begin{array}{l}
h_{\bar{A}_{1} \ldots \bar{A}_{r} D}(0)=0, \quad \forall A_{1}, \ldots A_{r}, D \in\{1, \ldots, n\}, r<\ell \\
h_{\bar{A}_{1}^{0} \ldots \bar{A}_{\ell}^{0} D^{0}}(0) \neq 0, \quad \text { for some } A_{1}^{0}, \ldots A_{r}^{0}, D^{0} \in\{1, \ldots, n\} .
\end{array}\right.
$$

If no such $\ell$ exists then we set $\ell_{0}=\infty$. Observe that if $M$ is $k$-nondegenerate at 0 for some $k$, then $\ell_{0} \leq k$, but $\ell_{0}<\infty$ does not imply finite nondegeneracy. Also, note 
that, for any $r \leq \ell_{0}$, the subspace $F_{r-1}(0)=\overline{\mathcal{V}}_{0}$ and, hence, the indices $a^{r}, b^{r}$, etc., introduced above run over the whole index set $\{1, \ldots, n\}$.

(Also note, by the fact that $L_{A}$ is adapted to the filtration (1.9), that if $\ell_{0}<\infty$ then we can take $D^{0}=1$ in (1.17).)

LEMMA 1.18. For any integer $r \geq 2$ and any integer $j \geq 0$ such that $j+r \leq \ell_{0}$ and indices $A_{1}, \ldots, A_{r}, C_{1} \ldots, C_{j}, D \in\{1, \ldots, n\}$, the following holds

$$
h_{\bar{A}_{1} \ldots \bar{A}_{r-1} \bar{A}_{r} D}(0)=\left(L_{\bar{A}_{r}} h_{\bar{A}_{1} \ldots \bar{A}_{r-1} D}\right)(0)
$$

$$
\left(L_{\bar{C}_{1}} \ldots L_{\bar{C}_{j}} h_{\bar{A}_{1} \ldots \bar{A}_{r-1} \bar{A}_{r} D}\right)(0)=\left(L_{\bar{C}_{1}} \ldots L_{\bar{C}_{j}} L_{\bar{A}_{r}} h_{\bar{A}_{1} \ldots \bar{A}_{r-1} D}\right)(0) .
$$

In particular,

$$
h_{\bar{A}_{1} \bar{A}_{2} \ldots \bar{A}_{\ell_{0} D}}(0)=\left(L_{\bar{A}_{\ell_{0}}} \ldots L_{\bar{A}_{2}} h_{\bar{A}_{1} D}\right)(0) .
$$

Proof. The first identity in (1.19) follows immediately by evaluating (1.14) at 0 and using the definition of $\ell_{0}$. In particular, it follows that

$$
\left(L_{\bar{A}_{r}} h_{\bar{A}_{1} \ldots \bar{A}_{r-1} D}\right)(0)=0
$$

for any $2 \leq r \leq \ell_{0}$. Now, the second identity in (1.19) follows by applying $L_{\bar{C}_{1}}$ to (1.14) and using (1.21). The conclusion of Lemma 1.18 follows by induction.

Recall that $M$ is said to be of finite type at $0 \in M$ if $L_{A}, L_{\bar{A}}$ and all their repeated commutators

$$
\left[X_{m},\left[X_{m-1}, \ldots\left[X_{2}, X_{1}\right] \ldots\right]\right], \quad X_{1}, \ldots, X_{m} \in\left\{L_{1}, \ldots, L_{n}, L_{\overline{1}}, \ldots, L_{\bar{n}}\right\},
$$

evaluated at 0 span $\mathbb{C} T_{0} M$. The commutator in (1.22) is said to have length $m$. (A commutator of length one is simply one of the vector fields $L_{A}, L_{\bar{A}}$.) If $M$ is of finite type at 0 , then it is said to be of type $m_{0}$ if $m_{0}$ is the smallest integer for which all commutators of the form (1.22) of lengths $\leq m_{0}$ span $\mathbb{C} T_{0} M$. Define $\ell_{1}$ to be the smallest integer $\ell$ for which

$$
\left\{\begin{array}{l}
\left\langle\theta,\left[L_{\bar{A}_{r}}, \ldots\left[L_{\bar{A}_{1}}, L_{D}\right] \ldots\right]\right\rangle(0)=0, \quad \forall A_{1}, \ldots A_{r}, D \in\{1, \ldots, n\}, r<\ell \\
\left\langle\theta,\left[L_{\bar{A}_{\ell}^{0}}, \ldots\left[L_{\bar{A}_{1}^{0}}, L_{D^{0}}\right] \ldots\right]\right\rangle(0) \neq 0, \quad \text { for some } A_{1}^{0}, \ldots A_{r}^{0}, D^{0} \in\{1, \ldots, n\} .
\end{array}\right.
$$

If no such $\ell$ exists then we set $\ell_{1}=\infty$. Observe that $\ell_{1}<\infty$ implies that $M$ is of finite type $m_{0} \leq \ell_{1}+1$ at 0 , but the converse is not true, i.e. $M$ can be of finite type at 0 while $\ell_{1}=\infty$.

Proposition 1.24. If either of the two integers $\ell_{0}, \ell_{1}$ is finite, then they are equal. Indeed, for any $r \leq \ell_{0}$, it holds that

$$
\left\langle\theta,\left[L_{\bar{A}_{r}}, \ldots\left[L_{\bar{A}_{1}}, L_{D}\right] \ldots\right]\right\rangle(0)=-h_{\bar{A}_{1} \ldots \bar{A}_{r} D}(0),
$$

for all $A_{1}, \ldots A_{r}, D \in\{1, \ldots, n\}$. In particular, if $M$ is $k$-nondegenerate at 0 , then it is also of finite type $\leq k+1$.

Proof. Note that the first part of Proposition 1.24 clearly follows from (1.25). Hence, we shall only prove (1.25). For any 1 -form $\xi$ and vector fields $X, Y$, we have 
the following well known identity (see e.g. [He])

$$
\langle d \xi, X \wedge Y\rangle=X\langle\xi, Y\rangle-Y\langle\xi, X\rangle-\langle\xi,[X, Y]\rangle .
$$

Thus, for a holomorphic 1-form $\omega$ on $M$, we obtain

$$
\left\langle\omega,\left[L_{\bar{A}}, L_{d}\right]\right\rangle=L_{\bar{A}}\left\langle\omega, L_{D}\right\rangle-\left\langle\mathcal{L}_{\bar{A}} \omega, L_{D}\right\rangle .
$$

By applying (1.27) with $\omega=\theta$, we deduce that

$$
\left\langle\theta,\left[L_{\bar{A}_{1}}, L_{D}\right]\right\rangle=-h_{\bar{A}_{1} D} .
$$

By Lemma 1.18 and the symmetry of the tensors $h_{\bar{A}_{1} \ldots \bar{A}_{r} D}(0)$, we then deduce that

$$
\left(L_{\bar{C}_{1}} \ldots L_{\bar{C}_{s}}\left\langle\theta,\left[L_{\bar{A}_{1}}, L_{D}\right]\right\rangle\right)(0)=-h_{\bar{C}_{1} \ldots \bar{C}_{s} \bar{A}_{1} D}(0), \quad \forall 0 \leq s \leq \ell_{0}-1,
$$

where $s=0$ in (1.28) means $\left\langle\theta,\left[L_{\bar{A}_{1}}, L_{D}\right]\right\rangle(0)=-h_{\bar{A}_{1} D}(0)$. By applying (1.27) with $\omega=\mathcal{L}_{\bar{B}_{j}} \ldots \mathcal{L}_{\bar{B}_{1}} \theta$, we obtain

$$
\left\langle\mathcal{L}_{\bar{B}_{j}} \ldots \mathcal{L}_{\bar{B}_{1}} \theta,\left[L_{\bar{A}_{1}}, L_{D}\right]\right\rangle=L_{\bar{A}_{1}} h_{\bar{B}_{1} \ldots \bar{B}_{j} D}-h_{\bar{A}_{1} \bar{B}_{1} \ldots \bar{B}_{j} D}
$$

Hence, it follows from Lemma 1.18 and the symmetry of the $h_{\bar{A}_{1} \ldots \bar{A}_{r} D}(0)$ that

$$
\left(L_{\bar{C}_{1}} \ldots L_{\bar{C}_{s}}\left\langle\mathcal{L}_{\bar{B}_{j}} \ldots \mathcal{L}_{\bar{B}_{1}} \theta,\left[L_{\bar{A}_{1}}, L_{D}\right]\right\rangle\right)(0)=0, \quad \forall 1 \leq j+s \leq \ell_{0}-1 .
$$

Now, assume that

$$
\begin{aligned}
\left(L_{\bar{C}_{1}} \ldots L_{\bar{C}_{s}}\left\langle\theta,\left[L_{\bar{A}_{r}}, \ldots\left[L_{\bar{A}_{1}}, L_{D}\right]\right\rangle\right)\right. & (0)= \\
& -h_{\bar{C}_{1} \ldots \bar{C}_{s} \bar{A}_{1} \ldots \bar{A}_{r} D}(0), \quad \forall 1 \leq s+r \leq \ell_{0},
\end{aligned}
$$

where $s \geq 0$ and the meaning for $s=0$ is analogous to (1.28), and

$$
\begin{aligned}
\left(L_{\bar{C}_{1}} \ldots L_{\bar{C}_{s}}\left\langle\mathcal{L}_{\bar{B}_{j}} \ldots \mathcal{L}_{\bar{B}_{1}} \theta,\left[L_{\bar{A}_{r}}, \ldots\left[L_{\bar{A}_{1}}, L_{D}\right] \ldots\right]\right\rangle\right)(0) & =0, \\
& \forall 1 \leq j+s+r \leq \ell_{0},
\end{aligned}
$$

where $j, s \geq 0$, for $r=1, \ldots R$. Observe that we have proved this for $R=1$. Now, if $R<\ell_{0}$, then the (1.31) and (1.32) follows for all $r=1, \ldots, R+1$ by applying (1.27) and Lemma 1.18. The verification of this is straightforward and left to the reader. By induction, we deduce that (1.31) and (1.32) hold for $r=1, \ldots, \ell_{0}$. In particular, (1.25) holds for any $r=1, \ldots, \ell_{0}$. This completes the proof of Proposition 1.24 .

So far, everything has been done with an arbitrary choice of basis $T, L_{A}, L_{\bar{A}}$, except that we chose the $L_{A}$ to be adapted to the filtration in (1.9) as explained above. We shall now use the fact that $M$ is embedded in $\mathbb{C}^{N}$ and choose a particular basis.

LEMMA 1.33. Let $M \subset \mathbb{C}^{N}$ be a smooth real hypersurface. Then, there is a basis $T, L_{A}, L_{\bar{A}}$ such that $T$ is real, the $L_{A}$ adapted to the filtration (1.9) as explained above, and

$$
R_{\bar{A} B}^{C} \equiv R_{D B}^{C} \equiv R_{\bar{A}}^{C} \equiv R_{B}^{C} \equiv 0
$$


for all indices $A, B, C, D \in\{1, \ldots, n\}$.

REMARK. Clearly, the conditions (1.34) are equivalent to $d \theta^{C}=0$. Based on this observation, an alternative proof of Lemma 1.33 can be given by pulling back suitable coordinate functions from the ambient space.

Proof. By making use of the identity (1.27), we conclude that

$$
\begin{array}{cl}
R_{\bar{A} B}^{C}=-\left\langle\theta^{C},\left[L_{\bar{A}}, L_{B}\right]\right\rangle, & R_{A B}^{C}=-\left\langle\theta^{C},\left[L_{A}, L_{B}\right]\right\rangle, \\
R_{\bar{A}}^{C}=-\left\langle\theta^{C},\left[L_{\bar{A}}, T\right]\right\rangle, & R_{A}^{C}=-\left\langle\theta^{C},\left[T, L_{A}\right]\right\rangle .
\end{array}
$$

Hence, to prove the lemma, it is equivalent to show that there is a basis $T, L_{A}, L_{\bar{A}}$ with $T$ real and $L_{A}$ adapted to the filtration (1.9) such that the $L_{A}$ commute, and $\left[L_{\bar{A}}, L_{B}\right]$ and $\left[L_{A}, T\right]$ are multiples of $T$. The existence of such a basis, disregarding the adaption of the $L_{A}$ to the filtration, is well known (see e.g. [BER3, Proposition 1.6.9]). Since the adaption of the $L_{A}$ is a condition only at the point 0 , we may achieve this by applying a linear transformation with constant coefficients to any basis $L_{A}$. Such a transformation does not affect any commutator relations and, hence, the lemma follows.

In what follows, we shall assume that (1.34) holds.

2. A Reflection Identity for CR Diffeomorphisms. Let $M$ be a smooth CR manifold as in the preceeding section, and let $\hat{M}$ be another smooth CR manifold of the same dimension and CR dimension, with distinguished point $\hat{0} \in \hat{M}$. We shall denote corresponding objects on $\hat{M}$ by using^; e.g. $\hat{\mathcal{V}} \subset \mathbb{C} T \hat{M}$ denotes the $\mathrm{CR}$ bundle on $\hat{M}, \hat{T}, \hat{L}_{A}, \hat{L}_{\bar{A}}$ is a basis for $C^{\infty}(\hat{U}, \mathbb{C} T \hat{M})$, where $\hat{U}$ is some sufficiently small neighborhood of $\hat{0} \in \hat{M}$. We shall assume that both $M$ and $\hat{M}$ are embeddable, locally near $0 \in M$ and $\hat{0} \in \hat{M}$, as real hypersurfaces in $\mathbb{C}^{N}$. Hence, (1.35) holds on $M$ and analogous identities on $\hat{M}$.

Assume that $f: M \rightarrow \hat{M}$ is a smooth CR diffeomorphism defined near 0 in $M$ such that $f(0)=\hat{0}$. Recall that a smooth mapping $f: M \rightarrow \hat{M}$ is called CR if $f_{*}\left(\mathcal{V}_{p}\right) \subset \hat{\mathcal{V}}_{f(p)}$, where $f_{*}: \mathbb{C} T M \rightarrow \mathbb{C} T \hat{M}$ denotes the tangent mapping or push forward, for every $p \in M$; a CR diffeomorphism is a diffeomorphism which is CR and whose inverse is also CR. In particular, if $f$ is a CR diffeomorphism then, for every $p \in M$ near $0, f_{*}\left(\mathcal{V}_{p}\right)=\hat{\mathcal{V}}_{f(p)}$. We introduce the smooth $G L\left(\mathbb{C}^{n}\right)$-valued function $\left(\gamma_{B}^{A}\right)$, and real-valued functions $\xi, \eta^{A}$ so that

$$
f_{*}\left(L_{B}\right)=\gamma_{B}^{A} \hat{L}_{A}, \quad f_{*}\left(L_{\bar{B}}\right)=\overline{\gamma_{B}^{A}} \hat{L}_{\bar{A}}, \quad f_{*}(T)=\xi \hat{T}+\eta^{A} \hat{L}_{A}+\overline{\eta^{A}} \hat{L}_{\bar{A}} .
$$

We can write (2.1) using matrix notation as

$$
f_{*}\left(T, L_{B}, L_{\bar{B}}\right)=\left(\hat{T}, \hat{L}_{A}, \hat{L}_{\bar{A}}\right)\left(\begin{array}{ccc}
\xi & 0 & 0 \\
\frac{\eta^{A}}{\eta^{A}} & \gamma_{B}^{A} & 0 \\
\hline \gamma_{B}^{A}
\end{array}\right) .
$$

By duality, we then have

$$
f^{*}\left(\begin{array}{c}
\hat{\theta} \\
\hat{\theta}^{A} \\
\hat{\theta}^{\bar{A}}
\end{array}\right)=\left(\begin{array}{ccc}
\xi & 0 & 0 \\
\frac{\eta^{A}}{\eta^{A}} & \gamma_{B}^{A} & 0 \\
\hline \gamma_{B}^{A}
\end{array}\right)\left(\begin{array}{c}
\theta \\
\theta^{B} \\
\theta^{\bar{B}}
\end{array}\right) .
$$

The main technical result in this section is the following, which can be viewed as reflection identities for $\gamma_{E}^{D}$ and $\eta^{D}$. 
THEOREM 2.4. If $\hat{M}$ is $k_{0}$-nondegenerate at $\hat{0} \in \hat{M}$, then the following identities holds for any indices $D, E \in\{1, \ldots, n\}$,

$$
\begin{aligned}
\gamma_{E}^{D} & =r_{E}^{D}\left(\overline{L^{J} \gamma_{A}^{C}}, \overline{L^{I} \xi} ; f\right), \\
\eta^{D} & =s^{D}\left(\overline{L^{J} \gamma_{A}^{C}}, \overline{L^{I} \xi} ; f\right)
\end{aligned}
$$

where

$$
r_{E}^{D}\left(\overline{L^{J} \gamma_{A}^{C}}, \overline{L^{I} \xi} ; q\right)(p), \quad s^{D}\left(\overline{L^{J} \gamma_{A}^{C}}, \overline{L^{I} \xi} ; q\right)(p)
$$

are smooth functions which are rational in $\overline{L^{J} \gamma_{A}^{C}}$ and polynomial in $\overline{L^{I} \xi}$, the indices $A, C$ run over the set $\{1, \ldots, n\}$, and $J, I$ over all multi-indices with $|J| \leq k_{0}-1$ and $|I| \leq k_{0}$; here, $(p, q) \in M \times \hat{M}$. Moreover, the functions in (2.7) depend only on $M$ and $\hat{M}$ (and not on the mapping $f$ ).

For the proof of Theorem 2.4, we shall make use of the following identity

$$
\left\langle d f^{*} \hat{\omega}, X \wedge Y\right\rangle=\left\langle d \hat{\omega}, f_{*} X \wedge f_{*} Y\right\rangle
$$

which holds for any 1-form $\hat{\omega}$ on $\hat{M}$ and vector fields $X, Y$ on $M$. First, letting $\hat{\omega}=\hat{\theta}$, $X=L_{\bar{A}}$, and $Y=L_{B}$, we obtain

$$
\xi h_{\bar{A} B}=\gamma_{B}^{D} \overline{\gamma_{A}^{C}} \hat{h}_{\bar{C} D}
$$

Here, and in what follows, we abuse the notation in the following way. For a function $\hat{c}$ defined on $\hat{M}$, we use the notation $\hat{c}$ to denote both the function $\hat{c} \circ f$ on $M$ and the function $\hat{c}$ on $\hat{M}$. It should be clear from the context which of the two functions is meant. For instance, in (2.9), we must have $\hat{h}_{\bar{C} D}=\hat{h}_{\bar{C} D} \circ f$. By letting $\hat{\omega}=\hat{\theta}^{E}$, $X=L_{\bar{A}}$, and $Y=L_{B}$ in (3.1), we obtain

$$
L_{\bar{A}} \gamma_{B}^{E}+\eta^{E} h_{\bar{A} B}=0 .
$$

Applying (2.8) with $X=L_{\bar{A}}, Y=T$, and $\hat{\omega}=\hat{\theta}$, we obtain

$$
L_{\bar{A}} \xi+\xi h_{\bar{A}}=\overline{\xi \gamma_{A}^{C}} \hat{h}_{\bar{C}}+\overline{\gamma_{A}^{C}} \eta^{D} \hat{h}_{\bar{C} D}
$$

and with $\hat{\omega}=\hat{\theta}^{C}$, we obtain

$$
L_{\bar{A}} \eta^{C}+\eta^{C} h_{\bar{A}}=0 .
$$

To obtain (2.11) and (2.12), we have used the fact

$$
\begin{aligned}
\left\langle d \hat{\omega}, \hat{L}_{\bar{C}} \wedge \hat{L}_{\bar{D}}\right\rangle & =\hat{L}_{\bar{C}}\left\langle\hat{\omega}, \hat{L}_{\bar{D}}\right\rangle-L_{\bar{D}}\left\langle\hat{\omega}, \hat{L}_{\bar{C}}\right\rangle-\left\langle\hat{\omega},\left[\hat{L}_{\bar{C}}, \hat{L}_{\bar{D}}\right]\right\rangle \\
& =0
\end{aligned}
$$

which holds for any holomorphic 1 -form $\hat{\omega}$ on $\hat{M}$ by the formal integrability of the CR bundle $\hat{\mathcal{V}}$. We apply (2.8) one last time, with $\hat{\omega}=\hat{\theta}^{C}, X=T$, and $Y=L_{A}$, to obtain

$$
T \gamma_{A}^{C}-L_{A} \eta^{C}-\eta^{C} \overline{h_{\bar{A}}}=0
$$


Lemma 2.14. For any nonnegative integer $k$, and indices $A_{1}, \ldots, A_{k}, B, C \in$ $\{1, \ldots, n\}$, the following identities hold

$$
L_{\bar{C}}\left(\gamma_{B}^{D} \hat{h}_{\bar{A}_{1} \ldots \bar{A}_{k} D}\right)=\gamma_{B}^{H} \overline{\gamma_{C}^{I}} \hat{h}_{\bar{A}_{1} \ldots \bar{A}_{k} \bar{I} H}-\gamma_{B}^{H} \overline{\gamma_{C}^{I}} \hat{h}_{\bar{A}_{1} \ldots \bar{A}_{k}} \hat{h}_{\bar{I} H}-\eta^{H} \hat{h}_{\bar{A}_{1} \ldots \bar{A}_{k} H} h_{\bar{C} B}
$$

and

$$
L_{\bar{C}}\left(\eta^{D} \hat{h}_{\bar{A}_{1} \ldots \bar{A}_{k} D}\right)=\eta^{H} \overline{\gamma_{C}^{I}} \hat{h}_{\bar{A}_{1} \ldots \bar{A}_{k} \bar{I} H}-\eta^{H} \bar{\gamma}_{C}^{I} \hat{h}_{\bar{A}_{1} \ldots \bar{A}_{k}} \hat{h}_{\bar{I} H}-\eta^{H} \hat{h}_{\bar{A}_{1} \ldots \bar{A}_{k} H} h_{\bar{C}}
$$

Proof. We shall prove (2.15). Recall that $\hat{h}_{\bar{A}_{1} \ldots \bar{A}_{k} D}$ in (2.15) denotes $\hat{h}_{\bar{A}_{1} \ldots \bar{A}_{k} D} \circ f$ by the convention introduced in $\S 1$. Hence,

$$
L_{\bar{C}}\left(\hat{h}_{\bar{A}_{1} \ldots \bar{A}_{k} D}\right)=\overline{\gamma_{C}^{I}}\left(\hat{L}_{\bar{I}} \hat{h}_{\bar{A}_{1} \ldots \bar{A}_{k} D}\right)
$$

where according to our convention $\hat{L}_{\bar{I}} \hat{h}_{\bar{A}_{1} \ldots \bar{A}_{k} D}=\left(\hat{L}_{\bar{I}} \hat{h}_{\bar{A}_{1} \ldots \bar{A}_{k} D}\right) \circ f$, and we obtain

$$
L_{\bar{C}}\left(\gamma_{B}^{D} \hat{h}_{\bar{A}_{1} \ldots \bar{A}_{k} D}\right)=\left(L_{\bar{C}} \gamma_{B}^{D}\right) \hat{h}_{\bar{A}_{1} \ldots \bar{A}_{k} D}+\gamma_{B}^{D} \overline{\gamma_{C}^{I}}\left(\hat{L}_{\bar{I}} \hat{h}_{\bar{A}_{1} \ldots \bar{A}_{k} D}\right)
$$

Let us rewrite $(2.10)$ as

$$
L_{\bar{A}} \gamma_{B}^{E}=-\eta^{E} h_{\bar{A} B}
$$

The identity (2.15) follows by substituting (2.19) in (2.18) and then applying Lemma 1.13 .

The proof of the identity (2.16) is completely analogous. Expand the left hand side by the chain rule, and then substitute for the derivatives of $\eta^{D}$ by using (2.12), and for the derivatives of $\hat{h}_{\bar{A}_{1} \ldots \bar{A}_{k} D}$ by using Lemma 1.13. The details are left to the reader.

The following two lemmas will be important in establishing Theorem 2.4.

Lemma 2.20. For any integer $k \geq 0$, and indices $A_{1}, \ldots, A_{k}, B \in\{1, \ldots, n\}$, the following identity holds

$$
\begin{aligned}
& \gamma_{B}^{D} \overline{\gamma_{A_{1}}^{C_{1}}} \ldots \overline{\gamma_{A_{k}}^{C_{k}}} \hat{h}_{\bar{C}_{1} \ldots \bar{C}_{k} D}=r_{\bar{A}_{1} \ldots \bar{A}_{k} B}\left(\overline{L^{J} \gamma_{A}^{C}} ; f\right)+\xi s_{\bar{A}_{1} \ldots \bar{A}_{k} B}\left(\overline{L^{J} \gamma_{A}^{C}} ; f\right) \\
& \quad+\sum_{l=1}^{k-1} \gamma_{E}^{D} \hat{h}_{\bar{C}_{1} \ldots \bar{C}_{l} D} t_{\bar{A}_{1} \ldots \bar{A}_{k} B}^{\bar{C}_{1} \ldots \bar{C}_{l} E}\left(\overline{L^{J} \gamma_{A}^{C}} ; f\right)+\sum_{l=1}^{k-1} \eta^{D} \hat{h}_{\bar{C}_{1} \ldots \bar{C}_{l} D} u_{\bar{A}_{1} \ldots \bar{A}_{k} B}^{\bar{C}_{1} \ldots \bar{C}_{l}}\left(\overline{L^{J} \gamma_{A}^{C}} ; f\right)
\end{aligned}
$$

where

$$
\begin{array}{ll}
r_{\bar{A}_{1} \ldots \bar{A}_{k} B}\left(\overline{L^{J} \gamma_{A}^{C}} ; q\right)(p), & s_{\bar{A}_{1} \ldots \bar{A}_{k} B}\left(\overline{L^{J} \gamma_{A}^{C}} ; q\right)(p), \\
t_{\bar{A}_{1} \ldots \bar{A}_{k} B}\left(\overline{L^{J} \gamma_{A}^{C}} ; q\right)(p), & u_{\bar{A}_{1} \ldots \bar{A}_{k} B}^{\bar{C}_{1} \ldots \bar{C}_{l} E}\left(\overline{L^{J} \gamma_{A}^{C}} ; q\right)(p)
\end{array}
$$

are polynomials in $\overline{L^{J} \gamma_{A}^{C}}$, where $A, C$ run over the indices $\{1, \ldots, n\}$ and $J=$ $\left(J_{1}, \ldots, J_{t}\right) \in\{1, \ldots n\}^{t}$ for $t \leq k-1$, whose coefficients are smooth functions of $(p, q) \in M \times \hat{M}$; here, we have used the notation $L^{J}=L_{J_{1}} \ldots L_{J_{t}}$. Moreover, the functions in (2.22) depend only on $M$ and $\hat{M}$ (and not on the mapping $f$ ).

Proof. We observe that (2.9) satisfies the conclusion of Lemma 2.20 for $k=1$. Assume that the conclusion of Lemma 2.20 holds for all integers $k=1, \ldots j-1$. Fix indices $A_{1}, \ldots, A_{j-1}, B \in\{1, \ldots, n\}$, choose an index $A_{j} \in\{1, \ldots, n\}$, and apply $L_{\bar{A}_{j}}$ to $(2.21)$ with $k=j-1$. The statement of the proposition for $k=j$ now follows by applying Lemma 2.14 and substituting for $L_{\bar{A}_{j}} \xi$ using (2.11). The proof is completed by induction on $k$. 
REMARK. In what follows, we shall use the notation $r, s, t$, and $u$ with varying sets of sub- and superscripts for "generic" functions which may be different from time to time.

Lemma 2.23. For any integer $k \geq 0$, and indices $A_{1}, \ldots, A_{k} \in\{1, \ldots, n\}$, the following identity holds

$$
\begin{aligned}
& \eta^{D} \overline{\gamma_{A_{1}}^{C_{1}}} \ldots \overline{\gamma_{A_{k}}^{C_{k}}} \hat{h}_{\bar{C}_{1} \ldots \bar{C}_{k} D}=r_{\bar{A}_{1} \ldots \bar{A}_{k}}\left(\overline{L^{J} \gamma_{A}^{C}}, L^{\bar{I}} \xi ; f\right) \\
& \quad+\sum_{l=1}^{k-1} \gamma_{E}^{D} \hat{h}_{\bar{C}_{1} \ldots \bar{C}_{l} D} t_{\bar{A}_{1} \ldots \bar{A}_{k}}^{\bar{C}_{1} \ldots \bar{C}_{l} E}\left(\overline{L^{J} \gamma_{A}^{C}} ; f\right)+\sum_{l=1}^{k-1} \eta^{D} \hat{h}_{\bar{C}_{1} \ldots \bar{C}_{l} D} u_{\bar{A}_{1} \ldots \bar{A}_{k}}^{\bar{C}_{1} \ldots \bar{C}_{l}}\left(\overline{L^{J} \gamma_{A}^{C}} ; f\right)
\end{aligned}
$$

where

$$
r_{\bar{A}_{1} \ldots \bar{A}_{k}}\left(\overline{L^{J} \gamma_{A}^{C}}, L^{\bar{I}} \xi ; q\right)(p), \quad u_{\bar{A}_{1} \ldots \bar{A}_{k}}^{\bar{C}_{1} \ldots \bar{C}_{l} E}\left(\overline{L^{J} \gamma_{A}^{C}} ; q\right)(p)
$$

are polynomials in $\overline{L^{J} \gamma_{A}^{C}}$ and $L^{\bar{I}} \xi$ in the former case and in $\overline{L^{J} \gamma_{A}^{C}}$ in the latter, where $A, C$ run over the indices $\{1, \ldots, n\}$ and $J=\left(J_{1}, \ldots, J_{t}\right), I=\left(I_{1}, \ldots, I_{t+1}\right)$, with $I_{i}, J_{j} \in\{1, \ldots n\}$, for $t \leq k-1$, whose coefficients are smooth functions of $(p, q) \in$ $M \times \hat{M}$; here, we have used the notation $L^{J}=L_{J_{1}} \ldots, L_{J_{t}}$ and $L^{\bar{J}}=L_{\bar{J}_{1}} \ldots, L_{\bar{J}_{t}}$. Moreover, the functions in (3.18) depend only on $M$ and $\hat{M}$ (and not on the mapping f).

Proof. We start with equation (2.11) and proceed as in the proof of Lemma 2.20. We leave the details to the reader.

We are ready to prove Theorem 2.4 .

Proof of Theorem 2.4. Using the fact that the matrices $\overline{\left(\gamma_{A}^{C}\right)}$ are invertible, we rewrite (2.21) and (2.24) as follows

$$
\begin{aligned}
& \gamma_{B}^{D} \hat{h}_{\bar{A}_{1} \ldots \bar{A}_{k} D}+\sum_{l=1}^{k-1} \gamma_{E}^{D} \hat{h}_{\bar{C}_{1} \ldots \bar{C}_{l} D} t_{\bar{A}_{1} \ldots \bar{A}_{k} B}^{\bar{C}_{1} \ldots \bar{C}_{l} E}\left(\overline{L^{J} \gamma_{A}^{C}} ; f\right)+ \\
& \sum_{l=1}^{k-1} \eta^{D} \hat{h}_{\bar{C}_{1} \ldots \bar{C}_{l} D}{ }^{\prime} u_{\bar{A}_{1} \ldots \bar{A}_{k B}}^{\bar{C}_{1} \ldots \bar{C}_{l}}\left(\overline{L^{J} \gamma_{A}^{C}} ; f\right)={ }^{\prime} r_{\bar{A}_{1} \ldots \bar{A}_{k} B}\left(\overline{L^{J} \gamma_{A}^{C}} ; f\right)+ \\
& { }^{\prime} s_{\bar{A}_{1} \ldots \bar{A}_{k} B}\left(\overline{L^{J} \gamma_{A}^{C}} ; f\right) \xi
\end{aligned}
$$

and

$$
\begin{aligned}
\eta^{D}\left(\hat{h}_{\bar{A}_{1} \ldots \bar{A}_{k} D}+\right. & \left.\sum_{l=1}^{k-1} \hat{h}_{\bar{C}_{1} \ldots \bar{C}_{l} D^{\prime}} u_{\bar{A}_{1} \ldots \bar{A}_{k}}^{\bar{C}_{1} \ldots \bar{C}_{l}}\left(\overline{L^{J} \gamma_{A}^{C}} ; f\right)\right)+ \\
& \sum_{l=1}^{k-1} \gamma_{F}^{D} \hat{h}_{\bar{C}_{1} \ldots \bar{C}_{l} D^{\prime}} t_{\bar{A}_{1} \ldots \bar{A}_{k}}^{\bar{C}_{1} \ldots \bar{C}_{l} F}\left(\overline{L^{J} \gamma_{A}^{C}} ; f\right)={ }^{\prime} r_{\bar{A}_{1} \ldots \bar{A}_{k}}\left(\overline{L^{J} \gamma_{A}^{C}}, \overline{L^{I} \xi} ; f\right)
\end{aligned}
$$

To prove the theorem, we must show that there are $n$ choices $\underline{A}^{j}$, where $\underline{A}^{j}=A_{1}^{j} \ldots A_{l_{j}}^{j}$ with $l_{j} \leq k_{0}$, such that the linear equations (2.26-2.27), with $\underline{A}=\underline{A}^{j}$ for $j=1, \ldots, n$ and $B=1, \ldots n$, can be solved uniquely for $\gamma_{B}^{D}$ and $\eta^{D}$ near 0 . For this it suffices to 
show that if, for some $\left(v_{B}^{D}, v^{D}\right) \in \mathbb{C}^{n^{2}+n}$,

$$
\begin{aligned}
v_{B}^{D} \hat{h}_{\bar{A}_{1} \ldots \bar{A}_{k} D}(0)+\sum_{l=1}^{k-1} v_{E}^{D} \hat{h}_{\bar{C}_{1} \ldots \bar{C}_{l} D}(0)^{\prime} t_{\bar{A}_{1} \ldots \bar{A}_{k} B}^{\bar{C}_{1} \ldots \bar{C}_{l} E}\left(\overline{L^{J} \gamma_{A}^{C}} ; f\right)(0)+ \\
\sum_{l=1}^{k-1} v^{D} \hat{h}_{\bar{C}_{1} \ldots \bar{C}_{l} D}(0)^{\prime} u_{\bar{A}_{1} \ldots \bar{A}_{k} B}^{\bar{C}_{1} \ldots \bar{C}_{l}}\left(\overline{L^{J} \gamma_{A}^{C}} ; f\right)(0)=0
\end{aligned}
$$

and

$$
\begin{array}{r}
v^{D}\left(\hat{h}_{\bar{A}_{1} \ldots \bar{A}_{k} D}(0)+\sum_{l=1}^{k-1} \hat{h}_{\bar{C}_{1} \ldots \bar{C}_{l} D}(0)^{\prime} u_{\bar{A}_{1} \ldots \bar{A}_{k}}^{\bar{C}_{1} \ldots \bar{C}_{l}}\left(\overline{L^{J} \gamma_{A}^{C}} ; f\right)(0)\right)+ \\
\sum_{l=1}^{k-1} v_{F}^{D} \hat{h}_{\bar{C}_{1} \ldots \bar{C}_{l} D}(0)^{\prime} t_{\bar{A}_{1} \ldots \bar{A}_{k}}^{\bar{C}_{1} \ldots \bar{C}_{l} F}\left(\overline{L^{J} \gamma_{A}^{C}} ; f\right)(0)=0,
\end{array}
$$

for all $A_{1}, \ldots A_{k}, B \in\{1, \ldots, n\}$ and all $k \leq k_{0}$, then $v_{B}^{D}=v^{D}=0$. To see this, note that (2.28-2.29), for $k=1$, implies directly that $v^{\alpha^{(2)}}=v_{B}^{\alpha^{(2)}}=0$; recall the convention introduced in $\S 1$ that the indices $\alpha^{(k+1)}$ run over $\left\{1, \ldots, r_{k}\right\}$, where $r_{k}=n-\operatorname{dim} F_{k}(0)$ as introduced in $\S 1$, and the indices $\left.a^{(k+1}\right)$ run over the set $\left\{r_{k}+1, \ldots n\right\}$. Thus, since $\hat{h}_{A a^{(2)}}(0)=0$, the equations $(2.28-2.29)$ for $k=2$ reduce to

$$
v_{B}^{a^{(2)}} \hat{h}_{\bar{A}_{1} \bar{A}_{2} a^{(2)}}(0)=0, \quad v^{a^{(2)}} \hat{h}_{\bar{A}_{1} \bar{A}_{2} a^{(2)}}(0)=0,
$$

which in turn implies $v^{\alpha^{(3)}}=v_{B}^{\alpha^{(3)}}=0$. Proceeding inductively, using at each step the fact that for any integers $1 \leq j<k \leq k_{0}$,

$$
\hat{h}_{\bar{A}_{1} \ldots \bar{A}_{j} a^{(k)}}(0)=0 \text {, }
$$

we conclude that the equations $(2.28-2.29)$, for $k \leq k_{0}$, imply $v^{\alpha^{\left(k_{0}+1\right)}}=v_{B}^{\alpha^{\left(k_{0}+1\right)}}=0$, which is equivalent to $v^{D}=v_{B}^{D}=0$ since $r_{k_{0}+1}=n$ for a $k_{0}$-nondegenerate $\mathrm{CR}$ manifold. This completes the proof of Theorem 2.4.

3. Proofs of Theorems 1, 2, and 3. We begin with the proof of Theorem 2 . For this proof, we shall need the following two lemmas. We shall keep the notation introduced in previous sections.

Lemma 3.1. For any indices $D, E, F \in\{1, \ldots, n\}$, multi-index $J$, and nonnegative integer $k$, we have the following

$$
\begin{aligned}
L_{E} \overline{L^{J} \gamma_{F}^{D}} & =r_{\bar{F} E}^{\bar{D} \bar{J}}\left(\overline{L^{I} \eta^{C}}\right), \\
L_{E} \overline{L^{J} T^{k} \eta^{D}} & =s_{E}^{\bar{D} \bar{J} k}\left(\overline{L^{I} T^{m} \eta^{C}}, \overline{L^{K} T^{m+1} \eta^{C}}\right),
\end{aligned}
$$

where the functions in (3.2-3) are smooth functions which are rational in the arguments appearing inside the parentheses. The indices $A, C$ run over the set $\{1, \ldots, n\}$, and $I, K$, over all multi-indices with $|I| \leq|J|,|K| \leq|J|-1$; the integer $m$ runs from 0 to $k$. Moreover, the functions in (3.2-3) depend only on $M$ and $\hat{M}$ (and not on the mapping $f$ ).

Proof. We shall use the following fact, which is an easy consequence of the commutator relations established in the proof of Lemma 1.32. For any vector field 
$X \in\left\{L_{A}, L_{\bar{A}}, T\right\}$ and any multi-index $J=\left(J_{1}, \ldots J_{|J|}\right)$, we have

$$
X L^{J}=L^{J} X+\sum_{|K| \leq|J|-1} c_{K} L^{K} T,
$$

where the $c_{K}$ are smooth functions on $M$ (which depend on $X$ and $J$ ). To prove (3.2), we observe that, in view of (3.4), we have

$$
\begin{aligned}
L_{E} \overline{L^{J} \gamma_{F}^{D}} & =\overline{L_{\bar{E}} L^{J} \gamma_{F}^{D}} \\
& =\overline{L^{J} L_{\bar{E}} \gamma_{F}^{D}}+\sum_{|K| \leq|J|-1} c_{K} \overline{L^{K} T \gamma_{F}^{D}} .
\end{aligned}
$$

The identity (3.2) follows from (2.10) and (2.13). The proof of (3.3) is similar, and left to the reader.

Lemma 3.6. For any index $E \in\{1, \ldots, n\}$, multi-indices $J$ and any nonnegative integer $k$, we have the following

$$
L_{E} \overline{L^{J} T^{k} \xi}=s_{E}^{\bar{J} k}\left(\overline{L^{K} \gamma_{A}^{C}}, \overline{L^{I} T^{m} \eta^{C}}, \overline{L^{I} T^{m} \xi}, \overline{L^{K} T^{m+1} \xi}, T^{m} \gamma_{A}^{C}, T^{m} \eta^{C} ; f\right)
$$

where the function in (3.7) is a smooth functions which are rational in the arguments preceding the ;. The indices $A, C$ run over the set $\{1, \ldots, n\}$, and $I, K$ over all multi-indices with $|I| \leq|J|,|K| \leq|J|-1$; the integer $m$ runs from 0 to $k$. Moreover, the function in (3.7) depends only on $M$ and $\hat{M}$ (and not on the mapping $f$ ).

Proof. We apply (3.4) as in the proof of Lemma 3.1 to deduce that that $L_{E} \overline{L^{J} T^{k} \xi}$ is linear in $\overline{L^{I} T^{m} \xi}, \overline{L^{K} T^{m+1} \xi}$, and $\overline{L^{I} T^{m} L_{\bar{E}} \xi}$. To evaluate the latter term, we make use of (2.11) and (2.13) to deduce that $\overline{L^{J} T^{k} L_{\bar{E}} \xi}$ is polynomial in $\overline{L^{I} T^{m} \xi}, \overline{L^{I} T^{m} \eta^{C}}$, $\overline{L^{K} \gamma_{A}^{C}}$, and $L^{\bar{I}} T^{m} \gamma_{A}^{C}$. Finally, we commute $L^{\bar{I}}$ and $T^{m}$ using (3.4), and then use (2.10) to conclude that $L^{\bar{I}} T^{m} \gamma_{A}^{C}$ is a linear function of $T^{m} \gamma_{A}^{C}$ and $T^{m} \eta^{C}$. Summing up, we obtain (3.7). This completes the proof of Lemma 3.6.

The following argument is inspired by the paper [Han2]. We shall say, for a function $u$ on $M$, that $u \in C_{p}^{a}$ if

$$
u=r\left(\overline{L^{I} \gamma_{A}^{C}}, \overline{L^{I} T^{m} \eta^{C}}, \overline{L^{I} T^{m} \xi}, L^{N} T^{n} \gamma_{A}^{C}, L^{N} T^{n} \eta^{C} ; f\right),
$$

where the function in (3.8) is a smooth functions which is rational in the arguments preceeding the ;. The indices $A, C$ run over the set $\{1, \ldots, n\}$. The multi-indices $I, N$ and the nonnegative integers $m, n$ run over all multi-indices with $|I|+m \leq p$, $|N|+n \leq a$. Moreover, the function in (3.8) should depend only on $M$ and $\hat{M}$ (and not on the mapping $f$ ). Similarly, we shall say that $u \in C_{p, q}^{a, b}$ if (3.8) holds with the additional condition that $m \leq q, n \leq b$. Observe that by Lemma 2.30 (and the reality of $\xi$ ), we have

$$
\gamma_{F}^{D}, \eta^{C}, \xi \in C_{k_{0}, 0}^{-1,-1}
$$

where the negative ones in the superscript signify that no terms involving $L^{N} \gamma_{A}^{C}$ or $L^{N} \eta^{C}$ appear. Recall that $k_{0}$ is the order of nondegeneracy of $\hat{M}$. By $(2.10-12)$, we obtain

$$
L^{\bar{J}} \gamma_{F}^{D}, L^{\bar{J}} \eta^{C} \in C_{k_{0}, 0}^{-1,-1}
$$


for any multi-index $J$. By applying $L_{E}$ to e.g. the equations for $L^{\bar{J}} \gamma_{F}^{D}$ and using Lemmas 3.1 and 3.6, we conclude that

$$
L_{E} L^{\bar{J}} \gamma_{F}^{D}=r_{E}^{\bar{S}}\left(\overline{L^{K} \gamma_{A}^{C}}, \overline{L^{I} T^{m} \eta^{C}}, \overline{L^{I} T^{m} \xi}, \gamma_{A}^{C}, \eta^{C} ; f\right)
$$

where $|K| \leq k_{0}-1,|I|+m \leq k_{0}$, and $m \leq 1$. By substituting for $\gamma_{A}^{C}$ and $\eta^{C}$ in (3.11) using the equations provided by (3.10), we conclude that $L_{E} \gamma_{F}^{D} \in C_{k_{0}, 1}^{-1,-1}$. We obtain a similar equation for $L_{E} L^{\bar{J}} \eta^{C}$. Hence, we obtain

$$
L_{E} L^{\bar{J}} \gamma_{F}^{D}, L_{E} L^{\bar{J}} \eta^{C} \in C_{k_{0}, 1}^{-1,-1}
$$

Next, by applying $L_{\bar{F}}$ to the equations for $L_{E} L^{\bar{J}} \gamma_{F}^{D}$ and $L_{E} L^{\bar{J}} \eta^{C}$, provided by (3.12), we obtain

$$
L_{\bar{F}} L_{E} L^{\bar{J}} \gamma_{F}^{D}, L_{\bar{F}} L_{E} L^{\bar{J}} \eta^{C} \in C_{k_{0}+1,1}^{-1,-1} .
$$

Similarly, repeated application of $L_{\bar{F}_{1}}, L_{\bar{F}_{2}}, \ldots, L_{\bar{F}_{k}}$ yields

$$
L_{\bar{F}_{k}} \ldots L_{\bar{F}_{1}} L_{E} L^{\bar{J}} \gamma_{G}^{D}, L_{\bar{F}_{k}} \ldots L_{\bar{F}_{1}} L_{E} L^{\bar{J}} \eta^{C} \in C_{k_{0}+k, 1}^{-1,-1} \text {. }
$$

Hence, by taking linear combinations of $L_{\bar{F}_{k}} \ldots L_{\bar{F}_{i}} L_{E} L_{\bar{F}_{i+1}} \ldots L_{\bar{F}_{k}} L^{\bar{J}}$, we deduce that

$$
\left[\ldots\left[L_{E}, L_{\bar{F}_{1}}\right], \ldots, L_{\bar{F}_{k}}\right] L^{\bar{J}} \gamma_{G}^{D},\left[\ldots\left[L_{E}, L_{\bar{F}_{1}}\right], \ldots, L_{\bar{F}_{k}}\right] L^{\bar{J}} \eta^{C} \in C_{k_{0}+k, 1}^{-1,-1}
$$

Let $\ell_{0} \leq k_{0}$ be the integer provided by Lemma 1.24 for which

$$
\left[\ldots\left[L_{E}, L_{\bar{F}_{1}}\right], \ldots, L_{\bar{F}_{k}}\right]=a T
$$

for some function $a$ with $a(0) \neq 0$. Then, (3.15) implies, in particular, that

$$
T \gamma_{G}^{C}, T \eta^{C} \in C_{k_{0}+\ell_{0}, 1}^{-1,-1} \text {. }
$$

Before proceeding, we shall need the following result on commutators of differential operators, which seems to be of independent interest.

Proposition 3.18. Let $\ell_{0}$ be the smallest integer for which (1.23) holds. Then, for any multi-index $J$, integer $k \geq 1$, and index $F \in\{1, \ldots, n\}$ there exist smooth functions $a^{E_{1} \ldots E_{m} \bar{F}_{1} \ldots \bar{F}_{s}}, b_{s}^{E_{1} \ldots E_{m}}$ such that

$$
\left.\sum_{m=1}^{|J|+k} \sum_{s=0}^{m \ell_{0}} a^{E_{1} \ldots E_{m} \bar{F}_{1} \ldots \bar{F}_{s}}\left[\ldots\left[L_{E_{1}} \ldots L_{E_{m}}, L_{\bar{F}_{1}}\right], L_{\bar{F}_{2}}\right] \ldots, L_{\bar{F}_{s}}\right]=L^{J} T^{k} .
$$

and

$$
\sum_{m=1}^{|J|+k} \sum_{s=0}^{k} b_{s}^{E_{1} \ldots E_{m}} \underbrace{\left.\left[\ldots\left[L_{E_{1}} \ldots L_{E_{m}}, L_{\bar{F}}\right], L_{\bar{F}}\right] \ldots, L_{\bar{F}}\right]}_{\text {length } s}=\left(h_{\bar{F} 1}\right)^{p} L^{J} T^{k}
$$

where $p=k+|J|-|J|_{1}+1$ and $|J|_{1}$ denotes the number of occurences of the index 1 in the multi-index $J$; here, the length of the commutator $\left.\left[\ldots\left[X, Y_{1}\right], Y_{2}\right] \ldots, Y_{s}\right]$ is $s$.

Proof. In this proof, we shall use the following notation to simplify the notation,

$$
C_{E_{1} \ldots E_{m}, \bar{F}_{1} \ldots \bar{F}_{s}}:=\left[\ldots\left[\left[L_{E_{1}} \ldots L_{E_{m}}, L_{\bar{F}_{1}}\right], L_{\bar{F}_{2}}\right] \ldots, L_{\bar{F}_{s}}\right]
$$


where $C_{E_{1} \ldots E_{m}}$ is understood to mean $L_{E_{1}} \ldots L_{E_{m}}$. Using bilinearity of the commutator and the identity

$$
[A B, C]=A[B, C]+[A, C] B
$$

a straightforward induction shows that

$$
\begin{aligned}
& {\left[\ldots\left[\left[L_{E_{1}} L_{E_{2}} \ldots L_{E_{m}}, L_{\bar{F}_{1}}\right], L_{\bar{F}_{2}}\right] \ldots, L_{\bar{F}_{s}}\right]=} \\
& \sum_{(\underline{i}, \underline{j}) \in P_{2}(s)} C_{E_{1}, \bar{F}_{i_{1}} \ldots \bar{F}_{i_{s-l}}} C_{E_{2} \ldots E_{m}, \bar{F}_{j_{1}} \ldots \bar{F}_{j_{l}}},
\end{aligned}
$$

where $P_{2}(s)$ denotes the set of all partitions of $\{1, \ldots, s\}$ into two disjoint increasing sequences $\underline{i}=\left(i_{1}, \ldots, i_{s-l}\right), 1 \leq i_{1}<\ldots i_{s-l} \leq s$, and $\underline{j}=\left(j_{1}, \ldots, j_{l}\right), 1 \leq j_{1}<$ $\ldots<j_{l} \leq s$ for $l=0, \ldots s$. (Of course, for e.g. $l=0$ the partition is understood to be the trivial one $\underline{i}=(1, \ldots, s)$ and $\underline{j}=\emptyset$.) Similarly, if we denote by $P_{m}(s)$ the set of all partitions of $\{1, \ldots, s\}$ into $m$ disjoint increasing sequences $\underline{i^{t}}=\left(i_{1}^{t}, \ldots, i_{s_{t}}^{t}\right)$, $1 \leq i_{1}^{t}<\ldots i_{s_{t}}^{t} \leq s, t=1, \ldots m$, and $\sum s_{t}=s$ (allowing empty sequences), then we have

$$
\begin{aligned}
& {\left[\ldots\left[\left[L_{E_{1}} L_{E_{2}} \ldots L_{E_{m}}, L_{\bar{F}_{1}}\right], L_{\bar{F}_{2}}\right] \ldots, L_{\bar{F}_{s}}\right]=} \\
& \quad \sum_{\left(\underline{i^{1}}, \ldots, \underline{i^{m}}\right) \in P_{m}(s)} C_{E_{1}, \bar{F}_{i_{1}^{1}} \ldots \bar{F}_{i_{s_{1}}}} \ldots C_{E_{m}, \bar{F}_{i_{1}^{m}} \ldots \bar{F}_{i_{m}^{m}}^{m}},
\end{aligned}
$$

Observe that $C_{E, \bar{F}_{1} \ldots \bar{F}_{s}}=a_{E, \bar{F}_{1} \ldots \bar{F}_{s}} T$, for some function $a_{E, \bar{F}_{1} \ldots \bar{F}_{s}}$ such that

$$
a_{E, \bar{F}_{1} \ldots \bar{F}_{s}}(0)=0, \quad \forall s<\ell_{0}, \quad \text { and } \quad a_{E, \bar{F}_{1} \ldots \bar{F}_{\ell_{0}}}(0)=h_{\bar{F}_{1} \ldots \bar{F}_{\ell_{0}} E}(0) \neq 0,
$$

for some choice of $F_{1}, \ldots, F_{\ell_{0}}$. Hence, with $s=\ell_{0}$ we obtain, by (3.23) and Lemma 1.24

$$
\begin{aligned}
{\left.\left[\ldots\left[L_{E_{1}} \ldots L_{E_{m}}, L_{\bar{F}_{1}}\right], L_{\bar{F}_{2}}\right] \ldots, L_{\bar{F}_{\ell_{0}}}\right]=\sum_{l=1}^{m}\left(h_{\bar{F}_{1} \ldots \bar{F}_{\ell_{0}} E_{l}}+o(1)\right) L_{E_{1}} \ldots \widehat{L_{E_{l}}} \ldots L_{E_{m}} T } & \\
& +\sum_{\substack{|K|+p=m \\
|K| \leq m-2}} b_{K p} L^{K} T^{p}+\sum_{|K| \leq m-2} c_{K} L^{K} T,
\end{aligned}
$$

where $b_{k p}(0)=0, \widehat{L_{E_{l}}}$ means that factor is omitted, and $o(1)$ denotes a function vanishing at 0 ; the last sum in (3.24) arises from arranging (by commuting) so that the vector field $T$ comes last in the first sum. Recall, from $\S 1$, that for each index $\alpha^{\left(\ell_{0}\right)} \in\left\{1, \ldots, r_{\ell_{0}}\right\}$ there exist $F_{1}, \ldots, F_{\ell_{0}}$ so that $h_{\bar{F}_{1} \ldots \bar{F}_{\ell_{0}} \alpha^{\left(\ell_{0}\right)}}(0) \neq 0$. For this argument, we only need the fact that there exist $F_{1}, \ldots, F_{\ell_{0}}$ so that $h_{\bar{F}_{1} \ldots \bar{F}_{\ell_{0} 1}}(0) \neq 0$. We choose $F_{1}, \ldots, F_{\ell_{0}}$ to be minimal, in the lexicographical ordering $\left(A_{1} \ldots A_{s}<\right.$ $B_{1} \ldots B_{s}$ if, for some $r \leq s, A_{i} \leq B_{i}$ for $i<r$, and $A_{r}<B_{r}$ ), with this property. Setting $E_{1}=\ldots E_{m}=1$, we observe that we can solve for $L_{1}^{m-1} T$ in (3.24). Setting $E_{1}=\ldots E_{m-1}=1$ and $L_{E_{m}}=L_{E}$ with $E \geq 2$, we can then solve for $L_{1}^{m-1} L_{E} T$. Proceeding inductively, we see that we can solve for any $L^{J} T$, with $|J|=m-1$, in terms of

$$
\left.\left[\ldots\left[L_{E_{1}} \ldots L_{E_{m}}, L_{\bar{F}_{1}}\right], L_{\bar{F}_{2}}\right] \ldots, L_{\bar{F}_{\ell_{0}}}\right], \quad b_{K p} L^{K} T^{p}, \quad L^{K} T
$$


where $K$ runs over multi-indices with $|K| \leq m-2$, and $p$ over positive integers such that $|K|+p=m$, and each $b_{k p}(0)=0$. Next, letting $s=2 \ell_{0}$ and $F_{\ell_{0}+l}=F_{l}$ for $l=1, \ldots \ell_{0}$, we obtain (by also using that $h_{\bar{F}_{1} \ldots \bar{F}_{s} E}$ is symmetric in the first $s$ indices $\left.F_{1}, \ldots F_{s}\right)$

$$
\begin{aligned}
& \left.\left[\ldots\left[L_{E_{1}} \ldots L_{E_{m}}, L_{\bar{F}_{1}}\right], L_{\bar{F}_{2}}\right] \ldots, L_{\bar{F}_{2 \ell_{0}}}\right]=\sum_{l=1}^{m} a_{E_{j} \bar{F}_{1} \ldots \bar{F}_{2 \ell_{0}}} L_{E_{1}} \ldots \widehat{L_{E_{l}}} \ldots L_{E_{m}} T \\
& +c_{\bar{F}} \sum_{1 \leq l_{1}<l_{2} \leq m}\left(h_{\bar{F}_{1} \ldots \bar{F}_{\ell_{0}} E_{l_{1}}} h_{\bar{F}_{1} \ldots \bar{F}_{\ell_{0}} E_{l_{2}}}+o(1)\right) L_{E_{1}} \ldots \widehat{L_{E_{l_{1}}}} \ldots \widehat{L_{E_{l_{2}}}} \ldots L_{E_{m}} T^{2} \\
& +\sum_{\substack{|K|+p=m \\
|K| \leq m-3}} o(1) L^{K} T^{p}+\sum_{\substack{|K|+p \leq m-1 \\
p=1,2}} c_{K p} L^{K} T^{p},
\end{aligned}
$$

where $c_{\vec{F}}$ is some combinatorial factor $(>0)$ which depends on the minimal set of indices $F_{1}, \ldots, F_{\ell_{0}}$. Using the fact that we have already solved for the $L^{J} T,|J|=$ $m-1$, in terms of $b_{K 2} L^{K} T^{2}$ where $b_{K 2}(0)=0$, a similar argument to the one used above shows that we can solve for each $L^{J} T^{2},|J|=m-2$, in terms of

$$
\left.\left[\ldots\left[L_{E_{1}} \ldots L_{E_{m}}, L_{\bar{F}_{1}}\right], L_{\bar{F}_{2}}\right] \ldots, L_{\bar{F}_{2 \ell_{0}}}\right], \quad o(1) L^{K} T^{p}, \quad L^{Q} T, \quad L^{K} T^{2}
$$

where $K, Q$, runs over multi-indices with $|K| \leq m-3,|Q| \leq m-2$, and $p$ over positive integers such that $|K|+p=m$. Proceeding by induction over $k$ (with the total order $m$ fixed), we eventually find that we can solve completely for $T^{m}$ in terms of

$$
\left.\left[\ldots\left[L_{E_{1}} \ldots L_{E_{m}}, L_{\bar{F}_{1}}\right], L_{\bar{F}_{2}}\right] \ldots, L_{\bar{F}_{m \ell_{0}}}\right], \quad L^{K} T^{p},
$$

with $|K|+p \leq m-1$. Substituting back, we obtain

$$
\begin{aligned}
\left.\sum_{n=0}^{k \ell_{0}} a^{E_{1} \ldots E_{m} \bar{F}_{1} \ldots \bar{F}_{n}}\left[\ldots\left[L_{E_{1}} \ldots L_{E_{m}}, L_{\bar{F}_{1}}\right], L_{\bar{F}_{2}}\right] \ldots, L_{\bar{F}_{n}}\right]=L^{J} T^{k} & \\
& +\sum_{|K|+p \leq m-1} c_{K p} L^{K} T^{p},
\end{aligned}
$$

where $m=|J|+k$. The proof of (3.19) is completed by a simple induction on the total degree $m$.

For the proof of (3.20), we proceed analogously by first setting $s=1$ and $E_{1}=$ $\ldots=E_{m}=1$ in (3.23). We find that

$$
m h_{\vec{F} 1} L_{1}^{m-1} T=C_{E_{1} \ldots E_{m}, \bar{F}}+\sum_{|K| \leq m-2} c_{K} L^{K} T .
$$

Next, with $E_{1}=\ldots=E_{m-1}=1$ and $E_{m}=E$, we obtain

$$
(m-1) h_{\bar{F} 1} L_{1}^{m-2} L_{E} T+h_{\bar{F} E} L_{1}^{m-1} T=C_{E_{1} \ldots E_{m}, \bar{F}}+\sum_{|K| \leq m-2} c_{K}^{\prime} L^{K} T .
$$

Thus, multiplying by $h_{\bar{F} 1}$ and using (3.26), we obtain (3.20) for a multi-index $J=$ $(1, \ldots, 1, E) \in\{1, \ldots, n\}^{m-1}$ and $k=1$. Similarly, we obtain (3.20) for arbitrary multi-indices $J$ and $k=1$. Proceeding inductively, setting $s=2,3, \ldots k$, using (3.23), and multiplying through by a suitable power of $h_{\bar{F} 1}$ to apply the results obtained in 
previous steps, we arrive at (3.20). The details are left to the reader. This completes the proof of Proposition 3.18.

To complete the proof of Theorem 2, let us observe the following schematic diagram which describes the action of applying the operators $L_{E}$ to elements in $C_{q+k, q}^{-1,-1}$

$$
\begin{aligned}
C_{q+k, q}^{-1,-1} \stackrel{L_{E_{1}}}{\longrightarrow} C_{q+k, q+1}^{q, q} \stackrel{L_{E_{2}}}{\longrightarrow} C_{q+k, q+2}^{q+1, q+1} \stackrel{L_{E_{3}}}{\longrightarrow} \ldots \stackrel{L_{E_{k}}}{\longrightarrow} \\
\stackrel{L_{E_{k}}}{\longrightarrow} C_{q+k, q+k}^{q+k-1, q+k-1} \stackrel{L_{E_{k+1}}}{\longrightarrow} C_{q+k, q+k}^{q+k, q+k} \stackrel{L_{E_{k+2}}}{\longrightarrow} C_{q+k, q+k}^{q+k+1, q+k} \stackrel{L_{E_{k+3}}}{\longrightarrow} \ldots
\end{aligned}
$$

The verification of the diagram is straightforward using Lemmas 3.1 and 3.6, and the details are left to the reader. Similarly, we have

$$
C_{p, q}^{a+k, a} \stackrel{L_{\bar{F}_{1}}}{\longrightarrow} C_{p+1, q}^{a+k, a+1} \stackrel{L_{\bar{F}_{2}}}{\longrightarrow} \ldots \stackrel{L_{\bar{F}_{k}}}{\longrightarrow} C_{p+k, q}^{a+k, a+k} \stackrel{L_{\bar{F}_{k+1}}}{\longrightarrow} C_{p+k+1, q}^{a+k, a+k} \stackrel{L_{\bar{F}_{k+2}}}{\longrightarrow} \ldots
$$

We claim that the following holds for any multi-index $J$ and nonnegative integer $k$,

$$
L^{J} T^{k} \gamma_{F}^{D}, L^{J} T^{k} \eta^{D} \in C_{k_{0}+m \ell_{0}, \min \left(k_{0}, m\right)}^{-1,-1}
$$

where $m=|J|+k$. Observe that (3.30) holds for $m=1$ by (3.12) and (3.17). We shall prove (3.30) by induction on $m$. Thus, assume that (3.30) holds for all $m \leq m_{0}-1$. By Proposition 3.18, we can produce the differential operator $L^{J} T^{k}$ by taking linear combinations of operators of the form $L^{\bar{P}} L^{Q} L^{\bar{R}}$, where $|P|+|R| \leq m_{0} \ell_{0}$, $|Q| \leq m_{0}$, and $m_{0}=|J|+k$. Applying first $L^{Q} L^{\bar{R}}$ to e.g. $\gamma_{F}^{D}$ we conclude, using (3.10) and the diagram (3.28), that $L^{Q} L^{\bar{R}} \gamma_{F}^{D} \in C_{k_{0}, \min \left(k_{0}, m_{0}\right)}^{m_{0}-1, \min \left(k_{0}, m_{0}-1\right)}$. By applying $L^{\bar{P}}$ to the equation for $L^{Q} L^{\bar{R}} \gamma_{F}^{D}$ and using the diagram (3.29), we obtain $L^{J} T^{k} \gamma_{F}^{D} \in$ $C_{k_{0}+m_{0} \ell_{0}, \min \left(k_{0}, m_{0}\right)}^{m_{0}-1, m_{0}-1}$. The conclusion $L^{J} T^{k} \gamma_{F}^{D} \in C_{k_{0}+m_{0} \ell_{0}, \min \left(k_{0}, m_{0}\right)}^{-1,-1}$ follows by using the induction hypothesis to substitute for the $L^{I} T^{m} \gamma_{A}^{C}$ and $L^{I} T^{m} \eta^{C}$, with $|I|+m \leq$ $m_{0}-1$, that appear in the equation for $L^{J} T^{k} \gamma_{F}^{D}$. By applying the same argument to $\eta^{D}$, we conclude that (3.30) holds for $m=m_{0}$ and, hence, for all $m$ by induction. This proves the claim. In particular, we then have

$$
T^{k} \gamma_{F}^{D}, T^{k} \eta^{D} \in C_{k_{0}+k \ell_{0}, \min \left(k_{0}, k\right)}^{-1,-1}
$$

By applying $L^{J}$ to these equations and using (3.28), we deduce that

$$
L^{J} T^{k} \gamma_{F}^{D}, L^{J} T^{k} \eta^{D} \in C_{k_{0}+k \ell_{0}, q(J, k)}^{a(J, k), q^{\prime}(J, k)}
$$

where

$$
\begin{aligned}
& a(J, k)=\min \left(k_{0}, k\right)+|J|-1 \\
& q(J, k)=\min \left(k_{0}+k \ell_{0}, \min \left(k_{0}, k\right)+|J|\right),
\end{aligned}
$$

and

$$
q^{\prime}(J, k)=\min \left(k_{0}+k \ell_{0}, \min \left(k_{0}, k\right)+|J|-1\right) .
$$

Observe that (3.32) implies

$$
L^{J} T^{k} \gamma_{F}^{D}, L^{J} T^{k} \eta^{D} \in C_{k_{0}+k_{0} \ell_{0}, k_{0}+k_{0} \ell_{0}}^{|J|+k-1, k_{0}+k_{0} \ell_{0}}, \quad \forall k: 0 \leq k \leq k_{0} .
$$

Now, from (3.4) it follows that $L^{R} T^{k} \xi=T^{k} L^{R} \xi$ modulo terms of the form $T^{k} L^{S} \xi$ with $|S|<|R|$. Hence, by applying $(2.11-13)$, we conclude that $L^{R} T^{k} \xi$ is a polynomial 
in $T^{m} \xi=\overline{T^{m} \xi}, \overline{T^{m} \eta^{C}}, L^{K} \gamma_{A}^{C}$, and $L^{I} T^{m-1} \eta^{C}$, where $m \leq k,|K| \leq|R|-1$, and $|I| \leq|R|$. It follows that we also have

$$
L^{J} T^{k} \xi \in C_{k_{0}+k_{0} \ell_{0}, k_{0}+k_{0} \ell_{0}}^{|J|+k-1, k_{0}+k_{0} \ell_{0}}, \quad \forall k: 0 \leq k \leq k_{0} .
$$

Let us introduce the new class $D_{p, q}^{a, b}$ consisting of functions $u$ for which there is an equation

$$
u=r\left(\overline{L^{I} \gamma_{A}^{C}}, \overline{L^{I} T^{m} \eta^{C}}, L^{N} T^{n} \xi, L^{N} T^{n} \gamma_{A}^{C}, L^{N} T^{n} \eta^{C} ; f\right),
$$

where $|I|+m \leq p, m \leq q,|N|+n \leq a, n \leq b$, and the function $r$ is rational in the arguments preceeding the ";" and only depends on $M$ and $\hat{M}$. By the above remarks concerning $L^{R} T^{k} \xi$ and (3.31), we have

$$
T^{k} \gamma_{F}^{D}, T^{k} \eta^{D} \in D_{k_{0}+k \ell_{0}, \min \left(k_{0}, k\right)}^{\min \left(k_{0}, k\right) \min \left(k_{0}, k\right)} .
$$

Since equations of the form (3.37) do not involve terms of the form $\overline{L^{I} T^{m} \xi}$, we obtain a different diagram describing the action of $L_{E}$ on the classes $D_{p, q}^{a, b}$, namely

$$
\begin{aligned}
D_{q+k, q}^{a, b} \stackrel{L_{E_{1}}}{\longrightarrow} D_{q+k, q+1}^{a+1, b} \stackrel{L_{E_{2}}}{\longrightarrow} \ldots & \stackrel{L_{E_{k}}}{\longrightarrow} D_{q+k, q+k}^{a+k-1, b} \stackrel{L_{E_{k+1}}}{\longrightarrow} \\
& \stackrel{L_{E_{k+1}}}{\longrightarrow} D_{q+k, q+k}^{a+k, b} \stackrel{L_{E_{k+2}}}{\longrightarrow} D_{q+k, q+k}^{a+k+1, b} \stackrel{L_{E_{k+3}}}{\longrightarrow} \ldots
\end{aligned}
$$

By (3.38-39), we deduce

$$
L^{J} T^{k} \gamma_{F}^{D}, L^{J} T^{k} \eta^{D} \in D_{k_{0}+k \ell_{0}, \min \left(k_{0}+k \ell_{0}, k_{0}+|J|\right)}^{k_{0}+|J| k_{0}}, \quad \forall k: k \geq k_{0}+1 .
$$

We have the following technical, but important, lemma.

Lemma 3.41. For any multi-index $J$, and nonnegative integer $k \leq k_{0}$, we have

$$
L^{J} T^{k} \gamma_{F}^{D}, L^{J} T^{k} \eta^{D}, L^{J} T^{k} \xi \in C_{k_{0}+\left(k_{0}+k_{0} \ell_{0}\right) \ell_{0}, k_{0}+\left(k_{0}+k_{0} \ell_{0}\right) \ell_{0}}^{-1,-1} .
$$

Proof. By (3.35-36), we have

$$
L^{J} T^{k} \gamma_{F}^{D}, L^{J} T^{k} \eta^{D}, L^{J} T^{k} \xi \in C_{k_{0}+k_{0} \ell_{0}, k_{0}+k_{0} \ell_{0}}^{|J|+k-1, k_{0}+\ell_{0} \ell_{0}} .
$$

Observe that (3.43) reduces the total order of the unconjugated terms by at least one. Now, in the equations given by (3.43), there may appear terms of the form $L^{I^{1}} T^{k_{1}} \gamma_{A}^{C}$, $L^{I^{1}} T^{k_{1}} \eta^{C}$, where $\left|I^{1}\right|+k_{1} \leq|J|+k-1$, and $k_{1} \leq k_{0}+k_{0} \ell_{0}$. For those term with $k_{0}+1 \leq k_{1} \leq k_{0}+k_{0} \ell_{0}$, we may apply (3.40) to deduce that

$$
L^{I^{1}} T^{k_{1}} \gamma_{A}^{C}, L^{I^{1}} T^{k_{1}} \eta^{C} \in D_{k_{0}+\left(k_{0}+k_{0} \ell_{0}\right) \ell_{0}, \min \left(k_{0}+\left(k_{0}+k_{0} \ell_{0}\right) \ell_{0}, k_{0}+\left|I^{1}\right|\right)}^{k_{0} \mid} .
$$

Note that, since $k_{1} \geq k_{0}+1$ and $\left|I^{1}\right|+k_{1} \leq|J|+k-1$, we have $k_{0}+\left|I^{1}\right| \leq|J|+k-2$. For those terms $L^{I^{1}} T^{k_{1}} \gamma_{A}^{C}, L^{I^{1}} T^{k_{1}} \eta^{C}$ with $k_{1} \leq k_{0}$, we may apply (3.35) again. In any case, we have reduced the total order of the unconjugated terms by two. In the equations given by (3.44), there may appear terms of the form $L^{I^{2}} T^{k_{2}} \gamma_{A}^{C}, L^{I^{2}} T^{k_{2}} \eta^{C}$, and also $L^{I^{2}} T^{k_{2}} \xi$, where $\left|I^{2}\right|+k_{2} \leq k_{0}+\left|I_{1}\right| \leq|J|+k-2$, and $k_{2} \leq k_{0}$. We again substitute for these terms, using the equations given by (3.35-36). This reduces the total order of the unconjugated terms another step. Proceeding in this way, alternately substituting using either (3.35-36) or (3.40), we eliminate all the unconjugated terms (in a finite number of steps). At each step we introduce new conjugated terms, but in 
view of (3.35-36) and (3.40), the total order of these never exceed $k_{0}+\left(k_{0}+k_{0} \ell_{0}\right) \ell_{0}$. This completes the proof of Lemma 3.41.

By substituting, using Lemma 3.41, for the conjugated terms that appear in the equations provided by (3.30), we conclude that for any multi-index $J$ and nonnegative integer $k$, we have

$$
L^{J} T^{k} \gamma_{F}^{D}, L^{J} T^{k} \eta^{D} \in D_{-1,-1}^{k_{0}+\left(k_{0}+k_{0} \ell_{0}\right) \ell_{0}, k_{0}+\left(k_{0}+k_{0} \ell_{0}\right) \ell_{0}} .
$$

By using (2.10) and (2.12), we conclude that for any multi-indices $R$ and $S$, any nonnegative integer $k$, and any indices $D, F \in\{1, \ldots n\}$, there are smooth functions, which are rational in their arguments preceeding the ";", such that

$$
\begin{aligned}
& L^{R} T^{k} L^{\bar{S}} \gamma_{F}^{D}=r^{R \bar{S} k}\left(L^{I} T^{j} \gamma_{A}^{C}, L^{I} T^{j} \eta^{C}, L^{I} T^{j} \xi ; f\right), \\
& L^{R} T^{k} L^{\bar{S}} \eta_{F}^{D}=s^{R \bar{S} k}\left(L^{I} T^{j} \gamma_{A}^{C}, L^{I} T^{j} \eta^{C}, L^{I} T^{j} \xi ; f\right),
\end{aligned}
$$

where $|I|+j \leq k_{0}+\left(k_{0}+k_{0} \ell_{0}\right) \ell_{0}$. Finally, by using (2.11), its complex conjugate, and Proposition 3.18, it is not difficult to see that $L^{R} T^{k} L^{\vec{S}} \xi$ can be expressed in terms of $\xi$ and derivatives of $\gamma_{A}^{C}, \overline{\gamma_{A}^{C}}, \eta^{C}$, and $\overline{\eta^{C}}$. Thus, in view of (3.46), we also have, for any $R, S$, and $k$,

$$
L^{R} T^{k} L^{\bar{S}} \xi=t^{R \bar{S} k}\left(L^{I} T^{j} \gamma_{A}^{C}, L^{I} T^{j} \eta^{C}, L^{I} T^{j} \xi, \overline{L^{I} T^{j} \gamma_{A}^{C}}, \overline{L^{I} T^{j} \eta^{C}}, \overline{L^{I} T^{j} \xi} ; f\right),
$$

where $I$ and $j$ run over the same indices as in (3.46). Now, recall that $\ell_{0} \leq k_{0}$. The conclusion of Theorem 2 follows by writing (3.46-47), for all $R, S, k$ such that

$$
|R|+|S|+k=k_{0}+\left(k_{0}+k_{0}^{2}\right) k_{0}+1
$$

in any coordinate systems $x=\left(x_{1}, \ldots, x_{2 n+1}\right)$ and $\hat{x}=\left(\hat{x}_{1}, \ldots \hat{x}_{2 n+1}\right)$ for $M$ and $\hat{M}$ near the points $0 \in M$ and $\hat{0} \in \hat{M}$, respectively, and observing that the same system of differential equations holds for any CR mapping $f$ sending a neighborhood of 0 in $M$ into $\hat{M}$ with $f(0)$ sufficiently close to $\hat{0}$. This completes the proof of Theorem 2 .

REMARK. We would like to point out that a much simpler conclusion of the proof of Theorem 2 can be given in the case $\ell_{0}=1$, i.e. when the Levi form of $M$ has at least one nonzero eigenvalue at 0 . We can then use the commutator identity (3.20) instead of (3.19) to conclude

$$
L^{J} T^{k} \gamma_{F}^{D}, L^{J} T^{k} \eta^{D} \in C_{k_{0}+k, \min \left(k_{0}, m\right)}^{-1,-1},
$$

instead of (3.30). By substituting for conjugated terms, using only (3.48), we obtain directly equations of the form (3.46) in which $|I|+j \leq 2 k_{0}$. We invite the reader to carry out the details in this case. Observe that the system of differential equations obtained for $f$ using this argument is of order $2 k_{0}+2$ rather than $k_{0}^{3}+k_{0}^{2}+2$ as given by Theorem 2 (or $k_{0}+\left(k_{0}+k_{0} \ell_{0}-1\right) \ell_{0}+2=3 k_{0}+1$ for $\ell_{0}=1$, which is the order that actually follows from the proof of Theorem 2 presented above).

A similar simpification of the proof in the general case would be possible if one could prove that it suffices to take the sum over $s$ in (3.19) to run from $s=0$ to $s=k \ell_{0}$ instead of all the way up to $s=m \ell_{0}$.

Proof of Theorem 1. The system of differential equations (0.3) is a so-called complete system of order $k_{0}^{3}+k_{0}^{2}+k_{0}+2$. In particular, any solution is completely determined by its $\left(k_{0}^{3}+k_{0}^{2}+k_{0}+1\right)$-jet at $0 \in M$ (see e.g. $\left[\mathrm{BCG}^{3}\right]$. cf. also $[\mathrm{Han} 2$, 
Proposition 2.2]). On the other hand, if $x \rightarrow Z(x)$ is an embedding of $M$ into $\mathbb{C}^{N}$ sending $p_{0} \in M$ to $0 \in \mathbb{C}^{N}$ and $x^{\prime} \rightarrow Z^{\prime}(x)$ is an embedding of $M^{\prime}$ sending $p_{0}^{\prime}$ to $0 \in \mathbb{C}^{N}$, then for any smooth CR mapping $f: M \rightarrow \hat{M}$, with $f\left(p_{0}\right)=p_{0}^{\prime}$, there exists (see e.g. [BER3, Proposition 1.7.14]) a formal power series mapping $Z^{\prime}=F(Z$ ), with $F(0)=0$, sending $M$ into $M^{\prime}$ (i.e. $\rho(Z, \bar{Z})$ divides $\rho^{\prime}(F(Z), \overline{F(Z)})$ in the ring of formal power series in $Z, \bar{Z}$; cf. e.g. [BER4]) such that

$$
Z^{\prime}(f(x)) \sim F(Z(x)),
$$

where $\sim$ denotes equality as formal power series. Also, by [BER4, Theorem 2.1.1], the $2 k_{0}$-jet at 0 of any invertible formal mapping $Z^{\prime}=F(Z)$, with $F(0)=(0)$, sending $M$ into $M^{\prime}$ determines the series $F(Z)$ completely. In particular, it follows from (3.49) that the $2 k_{0}$-jet at $p_{0}$ of a CR diffeomorphism $f: M \rightarrow M^{\prime}$, with $f\left(p_{0}\right)=p_{0}^{\prime}$, determines its $\left(k_{0}^{3}+k_{0}^{2}+k_{0}+1\right)$-jet at $p_{0}$. Hence, the conclusion of Theorem 1 follows from Theorem 2.

Proof of Theorem 3. We shall need the following proposition.

Proposition 3.50. If a smooth real hypersurface $M \subset \mathbb{C}^{N}$ is holomorphically nondegenerate at $p_{0} \in M$, then there exists an open neighborhood $U$ of $p_{0} \in M$ and a dense open subset $U^{\prime} \subset U$ such that $M$ is $(N-1)$-nondegenerate at every $p \in U^{\prime}$.

Proof. The statement that, under the hypotheses in the proposition, there exists an open neighborhood $U$ of $p_{0}$ such that $M$ is finitely nondegenerate on a dense open subset $U^{\prime \prime} \subset U$ is a consequence of [BER3, Theorem 11.7.5 (iii)]. To prove Proposition 3.50 , it suffices to show that if $M$ is not $k$-nondegenerate, for any $k \leq N-1$, on an open set $V$, then $M$ is in fact not finitely nondegenerate at any $p \in V$. Recall the subspaces $E_{j}(p) \subset T_{p}^{\prime} M$ defined for $j=0,1, \ldots$ in $\S 1$. Assume that $E_{N-1}(p)$ is a proper subspace of $T_{p}^{\prime} M$ for every $p \in V$, i.e. $M$ is not $k$-nondegenerate, for any $k \leq N-1$, in $V$. Since $\operatorname{dim}_{\mathbb{C}} T_{p}^{\prime} M=N$, we conclude, by (1.3), that there must be an open subset $V^{\prime} \subset V$ and an integer $1 \leq \ell \leq N-1$ such that

$$
E_{\ell-1}(q)=E_{\ell}(q), \quad \forall q \in V^{\prime} .
$$

We claim that if $E_{\ell-1}(q)=E_{\ell}(q)$ for all $q$ in some open sufficiently small set $V^{\prime} \subset M$, then in fact $E_{\ell-1}(q)=E_{k}(q)$ for all $k \geq \ell$ and all $q \in V^{\prime}$. To see this, observe that (3.51) implies that, for every $A_{1}, \ldots, \bar{A}_{\ell} \in\{1, \ldots, N\}$, there are smooth functions $a_{\bar{A}_{1} \ldots \bar{A}_{\ell}}^{\bar{C}_{1} \ldots \bar{C}_{j}}$ such that

$$
\mathcal{L}_{\bar{A}_{\ell}} \ldots \mathcal{L}_{\bar{A}_{1}} \theta=\sum_{j=0}^{\ell-1} a_{\bar{A}_{1} \ldots \bar{A}_{\ell}}^{\bar{C}_{1} \ldots \bar{C}_{j}} \mathcal{L}_{\bar{C}_{j}} \ldots \mathcal{L}_{\bar{C}_{1}} \theta
$$

in $V^{\prime}$. (3.52) implies that $E_{\ell+1}(q)=E_{\ell}(q)$ for $q \in V^{\prime}$, and the claim follows by induction. We conclude that $M$ is not finitely nondegenerate in $V^{\prime}$. A simple connectedness argument applied to each component of $V$ proves that $M$ cannot be finitely nondegenerate at any point in $V$. This completes the proof of Proposition 3.50.

We return to the proof of Theorem 3 . The fact that $M$ is minimal at $p_{0}$ implies, by a theorem of Trepreau (see e.g. [BER3, Theorem 8.1.1]; the analogous result in higher codimensions was proved by Tumanov), that for any open neighborhood $U$ of $p_{0}$ in $M$, there exists an open connected set $\Omega \subset \mathbb{C}^{N}$ (on "one side of $M$ ") such that $U^{\prime}:=$ $\bar{\Omega} \cap M \subset U$ is an open neighborhood of $p_{0}$ and every smooth CR function in $U$ is the smooth boundary value of a holomorphic function in $\Omega$. We deduce by the uniqueness 
of boundary values of holomorphic functions, Proposition 3.50, and Theorem 1 that there exists $p_{1} \in U^{\prime}$ such that if $f_{1}, f_{2}: U \rightarrow M^{\prime}$ are smooth CR diffeomorphisms into some smooth real hypersurface $M^{\prime} \subset \mathbb{C}^{N}$ and $\partial^{\alpha} f_{1}\left(p_{1}\right)=\partial^{\alpha} f_{2}\left(p_{1}\right)$, for all $|\alpha| \leq$ $2(N-1)$, then $f_{1}=f_{2}$ in $U^{\prime}$. Using this fact, the proof of Theorem 3 is completed exactly as the proof of [BER2, Theorem 2]. Choose $Y_{1}, \ldots, Y_{m} \in \operatorname{aut}\left(M, p_{0}\right)$ which are linearly independent over $\mathbb{R}$, and denote by $F(x, y)$, where $x=\left(x_{1}, \ldots, x_{2 N-1}\right)$ is some local coordinate system on $M$ near $p_{0}$ and $y=\left(y_{1}, \ldots, y_{m}\right) \in \mathbb{R}^{m}$, the time-one map of the flow $\exp t\left(y_{1} Y_{1}+\ldots+y_{m} Y_{m}\right)$, for $y$ in some sufficiently small neighborhood $V$ of the origin $\mathbb{R}^{m}$. The arguments in [BER2] combined with the uniqueness result stated above, for a suitably chosen open neighborhood of $U$ of $p_{0}$ in $M$, shows that the mapping $V \rightarrow J^{2(N-1)}\left(M, M^{\prime}\right)_{p_{1}}$, given by

$$
y \mapsto\left(\partial_{x}^{\alpha} F\left(p_{1}, y\right)\right)_{|\alpha| \leq 2(N-1)},
$$

is smooth and injective. Hence, $m \leq \operatorname{dim}_{\mathbb{R}} J^{2(N-1)}\left(M, M^{\prime}\right)_{p_{1}}$ which proves Theorem 3 .

Proof of Theorem 4. The conclusion of Theorem 4 is a direct consequence of Theorem 2 and Proposition 3.54 below. We shall use the notation $J^{k}\left(\mathbb{R}^{q}, \mathbb{R}^{m}\right)_{0}$ for the space of $k$-jets at $0 \in \mathbb{R}^{q}$ of smooth mappings $f: \mathbb{R}^{q} \rightarrow \mathbb{R}^{m}$, and $\lambda^{k}=\left(\lambda_{i}^{\beta}\right),|\beta| \leq k$ and $i=1, \ldots, m$, for the natural coordinates on this space in which the $k$-jet of $f$ is given by $\lambda_{i}^{\beta}=\partial_{x}^{\beta} f_{i}(0)$.

Proposition 3.54. Let $U \subset J^{k}\left(\mathbb{R}^{q}, \mathbb{R}^{m}\right)_{0} \times \mathbb{R}^{q}$ be an open domain. Let $r_{j}^{\alpha}\left(\lambda^{k}\right)(x)$, for any multi-index $\alpha \in \mathbb{Z}_{+}^{m}$ with $|\alpha|=k+1$ and any $j=1, \ldots, m$, be smooth $\left(C^{\infty}\right)$ functions on $U$. Then, for any $\lambda_{0}^{k} \in J^{k}\left(\mathbb{R}^{q}, \mathbb{R}^{m}\right)_{0}$ such that $\left(\lambda_{0}^{k}, 0\right) \in U$, there exists a uniquely determined smooth function $F: U_{0} \times V_{0} \rightarrow \mathbb{R}^{m}$, where $U_{0}$ is an open neighborhood of $\lambda_{0}^{k} \in J^{k}\left(\mathbb{R}^{q}, \mathbb{R}^{m}\right)_{0}$ and $V_{0}$ is an open neighborhood of $0 \in \mathbb{R}^{q}$, such that if $f=\left(f_{1}, \ldots, f_{m}\right)$ solves

$$
\partial_{x}^{\alpha} f_{j}=r_{j}^{\alpha}\left(\partial_{x}^{\beta} f\right), \quad \forall|\alpha|=k+1, j=1, \ldots, m
$$

near $0 \in \mathbb{R}^{q}$ and $j_{0}^{k}(f) \in U_{0}$, then

$$
F\left(j_{0}^{k}(f), \cdot\right)=f .
$$

REMARK 3.57. Observe that we do not claim that $F\left(\lambda^{k}, \cdot\right)$ solves (3.55) for any initial value $\lambda^{k}$, but only that if there is a solution with this initial condition then it coincides with $F\left(\lambda^{k}, \cdot\right)$. The idea for Proposition 3.54 was suggested to the author by D. Zaitsev.

Proof of Proposition 3.54. By a standard argument (considering the derivatives $\partial_{x}^{\beta} f,|\beta| \leq k$, as new unknowns), it suffices to prove Proposition 3.54 with $k=1$. Thus, we may assume that the system (3.55) is of the form

$$
\partial_{x_{j}} f_{i}=r_{i j}(f), \quad i=1, \ldots, m ; j=1, \ldots, q .
$$

Fix $\lambda_{0}^{1}$ as in the theorem. Write $x=\left(t, x^{\prime}\right) \in \mathbb{R} \times \mathbb{R}^{q-1}$ and consider the initial value problem for a system of ordinary differential equations

$$
\partial_{t} f_{i}(t, 0)=r_{i 1}(f(t, 0))(t, 0), \quad f(0,0)=\lambda^{1},
$$

for $\lambda^{1}$ in some sufficiently small neighborhood of $\lambda_{0}^{1}$. By a classical result (see [CL, Chapter 1.7], Theorem 7.5 and the following remarks), there is a smooth function 
$F^{1}: U_{1} \times V_{1} \rightarrow \mathbb{R}^{m}$, where $U_{1}$ is an open neighborhood of $\lambda_{0}^{1} \in J^{1}\left(\mathbb{R}^{q}, \mathbb{R}^{m}\right)_{0}$ and $V_{1}$ is an open neighborhood of $0 \in \mathbb{R}$, such that $t \mapsto F^{1}\left(\lambda^{1}, t\right)$ is the unique solution of (3.59). Next, write $x=\left(x_{1}, t, x^{\prime \prime}\right) \in \mathbb{R} \times \mathbb{R} \times \mathbb{R}^{q-2}$ and consider for each $x_{1} \in U_{1}$ the initial value problem

$$
\partial_{t} f_{i}\left(x_{1}, t, 0\right)=r_{i 1}\left(f\left(x_{1}, t, 0\right)\right)\left(x_{1}, t, 0\right), \quad f\left(x_{1}, 0,0\right)=F^{1}\left(\lambda^{1}, x_{1}\right) .
$$

Again by [CL, Chapter 1.7] (Theorem 7.5), there is a smooth function $F^{2}: U_{2} \times V_{2} \rightarrow$ $\mathbb{R}^{m}$, where $U_{2}$ is an open neighborhood of $\lambda_{0}^{1} \in J^{1}\left(\mathbb{R}^{q}, \mathbb{R}^{m}\right)_{0}$ and $V_{2}$ is an open neighborhood of $(0,0) \in \mathbb{R} \times \mathbb{R}$, such that $t \mapsto F^{2}\left(\lambda^{1}, x_{1}, t\right)$ is the unique solution of (3.60). Proceeding inductively in this way, we obtain the desired function $F$ after the $q$ :th step. The straightforward details are left to the reader. We emphasize however that the function so obtained need not be a solution of the system (3.58), but it satisfies $F\left(j_{0}^{1}(f), \cdot\right)=f$ whenever $f$ is a solution. This completes the proof of Proposition 3.54 .

The proof of Theorem 4 follows by applying Proposition 3.54 to the system of differential equations provided by Theorem 2 .

\section{Concluding Remarks.}

4.1. Abstract CR manifolds. In this paper, we have considered embedded real hypersurfaces as abstract manifolds with a(n integrable) CR structure. We have used the fact that the CR manifolds are embeddable (i.e. the CR structure is integrable) to choose a basis for the sections of $\mathbb{C} T M$ that satisfy certain commutation relations (Lemma 1.33). The author felt that the resulting equations (1.34) simplified the computations in the proofs to an extent which, by far, outweighed the loss of generality in assuming that the manifolds are embeddable. Without the use of the equations (1.34), the key equations (2.10-13) take the following form

$$
\begin{aligned}
& L_{\bar{A}} \gamma_{B}^{E}+\gamma_{D}^{E} R_{\bar{A} B}^{D}+\eta^{E} h_{\bar{A} B}=\gamma_{B}^{D} \overline{\gamma_{A}^{C}} \hat{R}_{\bar{C} D}^{E}, \\
& L_{\bar{A}} \xi+\xi h_{\bar{A}}=\xi \gamma_{A}^{C} \hat{h}_{\bar{C}}+\overline{\gamma_{A}^{C}} \eta^{D} \hat{h}_{\bar{C} D}, \\
& L_{\bar{A}} \eta^{C}+\eta^{C} h_{\bar{A}}+\gamma_{D}^{C} R_{\bar{A}}^{D}=\xi \gamma_{A}^{E} \hat{R}_{\bar{E}}^{C}+\overline{\gamma_{A}^{E}} \eta^{F} \hat{R}_{\bar{E} F}^{C}, \\
& T \gamma_{A}^{C}-L_{A} \eta^{C}+\gamma_{B}^{C} R_{A}^{B}-\eta^{C} \overline{h_{\bar{A}}}=\xi \gamma_{A}^{B} \hat{R}_{B}^{C}+\gamma_{A}^{B} \eta^{D} \hat{R}_{D B}^{C}+\gamma_{A}^{B} \overline{\eta^{E}} \hat{R}_{\bar{E} B}^{C} .
\end{aligned}
$$

Analogous reflection formulae to those in Theorem 2.4, as well as analogues of the crucial Lemmas 3.1, 3.6, and Proposition 3.18, can be established (with considerably more work than above). The author is confident that a proof of Theorem 2 for abstract CR manifolds (of hypersurface type) $M$ and $M^{\prime}$ of the same dimension can be produced from these ingredients, but he has not had the patience to check the details.

4.2. Infinitesimal CR automorphisms. It is clear from the proof of Theorem 3 above that in order for the estimate (0.5) to hold, it suffices that $M$ is minimal at $p_{0}$ and that there exists an open subset $U \subset M$ with $p_{0}$ in its boundary such that $M$ is finitely nondegenerate on $U$. The latter holds, in particular, if $M$ is holomorphically nondegenerate at $p_{0}$ (and, in the real-analytic case, only if), but may hold, in the case of merely smooth manifolds, even if $M$ is holomorphically degenerate at $p_{0}$ (see [BER3, Example 11.7.29]). On the other hand, as is mentioned in the introduction, if there exists a vector field $Y$ of the form (0.4), where the restrictions of the $a_{j}$ to $M$ are CR functions, which is tangent to $M$ near $p_{0}$, then $\operatorname{dim}_{\mathbb{R}} \operatorname{aut}\left(M, p_{0}\right)=\infty$. Let us 
call the restriction to $M$ of such a vector field $Y$ a $C R$ holomorphic vector field. Thus, one is led to the following question. Suppose $M$ is not finitely nondegenerate at any point in an open neighboorhood of $p_{0}$. Does there then exist a CR holomorphic vector field on $M$ near $p_{0}$ ? The author does not know, the answer to this question in general, but it seems to be related to the range of the tangential Cauchy-Riemann operator $\bar{\partial}_{b}$ (see e.g. [B] for the definition). We conclude this paper by briefly outlining this connection.

First, observe that a vector field $Y$ is CR holomorphic if and only if $Y$ is a section of $\overline{\mathcal{V}}$ and $[\bar{L}, Y]$ is a $\mathrm{CR}$ vector field (i.e. a section of $\mathcal{V}$ ) for every $\mathrm{CR}$ vector field $\bar{L}$. Now, suppose that there is an open set $U \subset M$ in which $M$ is not finitely nondegenerate at any point. We claim that there exists a (non-vanishing) $\mathrm{CR}$ holomorphic vector field $Y$ near $p \in U$ if (i) $\operatorname{dim}_{\mathbb{C}} E_{N-1}(q)$ (which is $<N$ for $q$ in $U$ by assumption) is maximal at $q=p$, and (ii) $\bar{\partial}_{b} u=f$ is solvable at $p$ for every $(0,1)$-form $f$ with $\bar{\partial}_{b} f=0$. For simplicity, we shall indicate the proof of this only in the case $\operatorname{dim}_{\mathbb{R}} E_{N-1}(p)=N-1$. We choose a smooth nonvanishing section $X$ of $\overline{\mathcal{V}}$ near $p$ such that $X(q) \in E_{k}(q)^{\perp}$ for all $k$ and all $q$ in an open neighborhood of $p$. (This can be done by assumption (i) above.) We denote by $L_{\overline{1}}, \ldots, L_{\bar{n}}$ a basis of the CR vector fields on $M$ near $p$, where $L_{n}:=\bar{L}_{\bar{n}}=X$. We choose a tranversal vector field $T$, as in $\S 1$, and denote by $\theta, \theta^{A}, \bar{\theta}^{\bar{A}}$, with notation and conventions as in $\S 1$, a dual basis of $T, L_{A}, L_{\bar{A}}$. The fact that $L_{n}$ is valued in $E_{k}^{\perp}$, for every $k$, implies that $\left[L_{\bar{A}}, L_{n}\right]=b_{\bar{A}} L_{n}$ modulo the $\mathrm{CR}$ vector fields. We shall look for a CR holomorphic vector field $Y$ of the form $u L_{n}$, where $u$ is a function to be determined. It is easy to see that $\left[L_{\bar{A}}, Y\right]$ is a CR vector field if and only if

$$
L_{\bar{A}} u+u b_{\bar{A}}=0
$$

and, hence, $Y$ is CR holomorphic if and only if (4.2.1) is satisfied for every $\bullet A \in$ $\{1, \ldots, n\}$. If we could solve

$$
\bar{\partial}_{b} v=f
$$

where $f=b_{\bar{A}} \theta^{\bar{A}}$, then $u=e^{-v}$ would solve (4.2.1). The $(0,1)$-form $f$ coincides with the form $\left.L_{n}\right\lrcorner \bar{\partial}_{b} \theta^{n}$, as the reader can verify. From this observation, one can check that $f$ satisfies the necessary compatibility condition for solvability,

$$
\left.\bar{\partial}_{b} f=\bar{\partial}_{b}\left(L_{n}\right\lrcorner \bar{\partial}_{b} \theta^{n}\right)=0 .
$$

Hence, if the tangential Cauchy-Riemann complex is solvable at level $(0,1)$ at $p$, then we can solve (4.2.2) and obtain a CR holomorphic vector field $Y=u L_{n}$ near $p$. However, the author knows of no results on solvability which apply in this situation (unless, of course, $M$ is real-analytic).

\section{REFERENCES}

[ABR] S. Alinhac, M. S. BAouendi, AND L. P. Rothschild, Unique continuation and regularity at the boundary for holomorphic functions, Duke Math. J., 61 (1990), pp. 635-653.

[BER1] M. S. BAouendi, P. Ebenfelt, And L. P. Rothschild, Parametrization of local biholomorphisms of real-analytic hypersurfaces, Asian J. Math., 1 (1997), pp. 1-16.

[BER2] M. S. BAOUENDI, P. EBEnfElt, AND L. P. Rothschild, CR automorphisms of real analytic CR manifolds in complex space, Comm. Anal. Geom., 6 (1998), pp. 291-315. 
[BER3] M. S. BAouendi, P. Ebenfelt, And L. P. Rothschild, Real submanifolds in complex space and their mappings, Princeton University Press, Princeton Math. Ser. 47, Princeton, NJ, 1999.

[BER4] M. S. BAouendi, P. Ebenfelt, And L. P. Rothschild, Rational dependence of smooth and analytic CR mappings on their jets, Math. Ann., 315 (1999), pp. 205-249.

[BER5] M. S. Baouendi, P. Ebenfelt, and L. P. Rothschild, Convergence and finite determination of formal CR mappings, J. Amer. Math. Soc., 13 (2000), pp. 697-723.

[BER6] M. S. BAouendi, P. EBenfelt, AND L. P. Rothschild, Local geometric properties of real submanifolds in complex space, Bull. Amer. Math. Soc., 37 (2000), pp. 309-336.

[BR] M. S. BAouendi AND L. P. Rothschild, A generalized complex Hopf Lemma and its application to CR mappings, Invent. Math., 111 (1993), pp. 331-148.

[BL] S. Bell And L. Lempert, A $C^{\infty}$ Schwarz reflection principle in one and several complex variables, J. Diff. Geom., 32 (1990), pp. 899-915.

[B] A. Boggess, CR manifolds and the tangential $C R$ complex, CRC Press, Inc., Boca Roaton, Fla., 1991.

[BCG $\left.{ }^{3}\right]$ R. L. Bryant, S. S. Chern, R. B. Gardner, H. L. Goldschmidt, P. A. Griffiths, Exterior Differential Systems, Mathematical Sciences Research Institute Publications, 18. Springer-Verlag, New York, 1991.

[ECa1] E. CARTAN, Sur la géométrie pseudo-conforme des hypersurfaces de deux variables complexes, I, Ann. Math. Pura Appl., 11 (1932), pp. 17-90, (or Oeuvres II, 1231-1304).

[ECa2] E. CARTAN, Sur la géométrie pseudo-conforme des hypersurfaces de deux variables complexes, II, Ann. Scoula Norm. Sup. Pisa, 1 (1932), pp. 333-354, (or Oeuvres III, 12171238).

[HCa] H. Cartan, Sur les groupes de transformations analytiques, Act. Sci. et Int., Hermann, Paris, 1935.

[CM] S. S. Chern And J.K. Moser, Real hypersurfaces in complex manifolds, Acta Math., 133 (1974), pp. 219-271.

[CR] E. M. ChIRKA AND C. ReA, Normal and Tangent ranks of CR mappings, Duke Math. J., 76 (1994), pp. 417-431.

[CL] E. A. Coddington and N. Levinson, Theory of ordinary differential equations, McGrawHill Book Co., Inc, New York, 1955.

[E1] P. EBENFELT, On the unique continuation problem for CR mappings into nonminimal hypersurfaces, J. Geom. Anal., 6 (1996), pp. 385-405.

[E2] P. Ebenfelt, Nondegeneracy conditions and normal forms for real hypersurfaces in complex space, in Journées "Équations aux Dérivées Partielles" (Saint-Jean-de-Monts, 1997), École Polytech., Palaiseau.

[E3] P. EBenfelt, New invariant tensors in CR structures and a normal form for real hypersurfaces at a generic Levi degeneracy, J. Differential Geom., 50 (1998), pp. 207-247.

[G] R. B. Gardner, The Method of Equivalence and Its Applications, SIAM CBMS-NSF Regional Conference Series in Applied Mathematics, Philadelphia, 1989.

[Han1] C. K. HAN, Analyticity of CR equivalences between real hypersurfaces in $\mathbb{C}^{n}$ with degenerate Levi form, Invent. Math., 73 (1983), pp. 51-69.

[Han2] C. K. HaN, Complete system for the mappings of CR manifolds of nondegenerate Levi forms, Math. Ann., 309 (1997), pp. 401-409.

[Hay] A. Hayashimoto, On the complete system of finite order for $C R$ mappings and its application, Osaka J. Math., 35 (1998), pp. 617-628.

[He] S. Helgason, Differential Geometry and Symmetric Spaces, Academic Press, New York and London, 1962.

[HK] X. Huang and S. G. Krantz, A unique continuation problem for holomorphic mappings, Comm. Part. Diff. Eq., 18 (1993), pp. 241- 263.

[K] S. KоваyAShi, Transformation Groups in Differential Geometry, Springer-Verlag, Berlin, 1972.

[KN] S. Koваyashi, K. Nomizu, Foundations of Differential Geometry, Volume I and II, Interscience Publ., New York, 1963.

[L] B. LAMEL, Holomorphic maps of real submanifolds in complex spaces of different dimensions, preprint (1999).

[S1] N. Stanton, Infinitesimal CR automorphisms of rigid hypersurfaces, Amer. J. Math., 117 
(1995), pp. 141-167.

[S2] N. Stanton, Infinitesimal CR automorphisms of real hypersurfaces, Amer. J. Math., 118 (1996), pp. 209-233.

[T1] N. TANAKA, On the pseudo-conformal geometry of hypersurfaces of the space of $n$ complex variables, J. Math. Soc. Japan, 14 (1962), pp. 397-429.

[T2] N. TANAKA, On generalized graded Lie algebras and geometric structures. I, J. Math. Soc. Japan, 19 (1967), pp. 215-254, (erratum 36, p. 1568).

[Z] D. ZAITSEV, Germs of local automorphisms of real-analytic CR structures and analytic dependence on $k$-jets, Math. Research Lett., 4 (1997), pp. 823-842. 